Cite this: Chem. Soc. Rev., 2019, 48,3842

Received 26th October 2018

DOI: $10.1039 / \mathrm{c} 8 \mathrm{cs} 00853 a$

rsc.li/chem-soc-rev

\section{Imperfections and their passivation in halide perovskite solar cells}

\author{
Bo Chen, ${ }^{a}$ Peter N. Rudd, ${ }^{a}$ Shuang Yang, (D) ab Yongbo Yuan ${ }^{c}$ and \\ Jinsong Huang (iD *ab
}

\begin{abstract}
All highly-efficient organic-inorganic halide perovskite (OIHP) solar cells to date are made of polycrystalline perovskite films which contain a high density of defects, including point and extended imperfections. The imperfections in OIHP materials play an important role in the process of charge recombination and ion migration in perovskite solar cells (PSC), which heavily influences the resulting device energy conversion efficiency and stability. Here we review the recent advances in passivation of imperfections and suppressing ion migration to achieve improved efficiency and highly stable perovskite solar cells. Due to the ionic nature of OIHP materials, the defects in the photoactive films are inevitably electrically charged. The deep level traps induced by particular charged defects in OIHP films are major non-radiative recombination centers; passivation by coordinate bonding, ionic bonding, or chemical conversion have proven effective in mitigating the negative impacts of these deep traps. Shallow level charge traps themselves may contribute little to nonradiative recombination, but the migration of charged shallow level traps in OIHP films results in unfavorable band bending, interfacial reactions, and phase segregation, influencing the carrier extraction efficiency. Finally, the impact of defects and ion migration on the stability of perovskite solar cells is described.
\end{abstract}

\section{Introduction}

The power conversion efficiency of solar cells based on organicinorganic halide perovskites has soared in recent years to attain

\footnotetext{
${ }^{a}$ Department of Applied Physical Sciences, The University of North Carolina at Chapel Hill, Chapel Hill, North Carolina 27599, USA. E-mail: jhuang@unc.edu

${ }^{b}$ Department of Mechanical and Materials Engineering,

University of Nebraska-Lincoln, Lincoln, Nebraska 68588, USA

${ }^{c}$ School of Physics \& Electronics, Hunan Key Laboratory of Super Microstructure \& Ultrafast Process, Central South University, Changsha, Hunan, 410083, China
}

a certified PCE of $24.2 \% .^{1-5}$ The impressive photovoltaic performance of perovskite solar cells (PSCs) benefits from the superior light absorption coefficient $\left(\sim 10^{5} \mathrm{~cm}^{-1}\right)$, long carrier diffusion lengths in polycrystalline films $(>1 \mu \mathrm{m})$, and the generation of free charges by photoexcitation of OIHP materials. ${ }^{6,7}$ The solution processable fabrication and affordable material cost enable a low levelized cost of electricity (LCOE) from perovskite photovoltaic modules with technoeconomic analysis showing LCOE within 0.05 USD per $\mathrm{kW} \mathrm{h}^{-1}$ if the module lifetimes can reach $15-20$ years. ${ }^{8,9}$ The tunability of the bandgap for perovskite photoactive layers from $1.2 \mathrm{eV}$ to

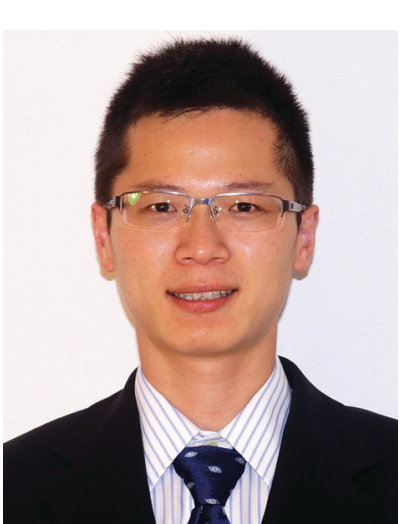

Bo Chen
Dr Bo Chen received his $B S$ in Physics from Zhejiang University in 2007, and his PhD degree in Materials Science and Engineering from Virginia Tech in 2012. Now he works as a postdoctoral research associate in Department of Applied Physical Sciences at University of North Carolina at Chapel Hill. His recent research focuses on physical properties of perovskite materials, perovskite solar cells, and perovskite/silicon tandem cells.

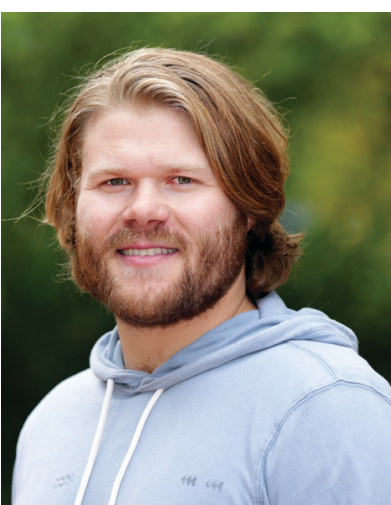

Peter N. Rudd received his BS in Chemistry from Denison University in 2017. He is currently a graduate student at The University of North Carolina at Chapel Hill. His research interests mainly focus on improving the efficiency and stability of perovskite based photovoltaic devices.
Peter N. Rudd 
$3.0 \mathrm{eV}$ by composition engineering enables the fabrication of perovskite/silicon tandem solar cells and perovskite/perovskite tandem solar cells with a theoretical efficiency limit beyond $30 \%,{ }^{10-15}$ far surpassing the Shockley-Queisser efficiency limit of single-junction solar cells. ${ }^{16}$

The efficiency of PSCs is determined by the recombination processes of photo-excited charge carriers. ${ }^{17-25}$ The charge carriers can recombine radiatively or non-radiatively, where the energy of recombined excess electrons and holes is eventually transferred as either a photon or phonon, respectively. ${ }^{23}$ Perovskite materials are reported to have high radiative recombination. Perovskite solar cell research stemmed from dye-sensitized solar cells in which surface defects induced recombination in light absorber (dyes) were generally ignored. Several initial studies done by Y. Shao et al. and Abate et al. discovered that imperfections in

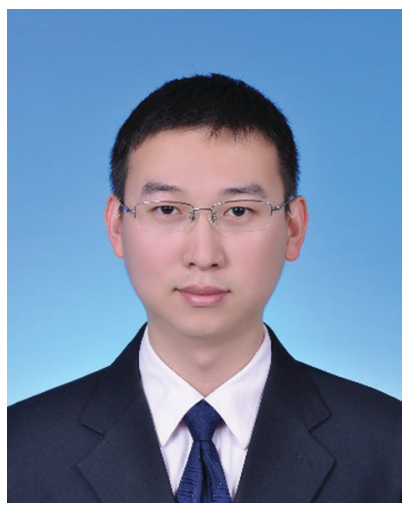

Shuang Yang try and physics of semiconducting materials and photo-electric devices.

Dr Shuang Yang received his Bachelor degree from Tsingtao University of Science \& Technology in 2011 and completed his PhD in 2016 at East China University of Science \& Technology. He then conducted postdoctoral research at Nanyang Technological University, University of Nesbraka-Lincoln and University of North CarolinaChapel Hill. Currently he is a professor at Shandong University. His research focus on the chemis-

Yongbo Yuan

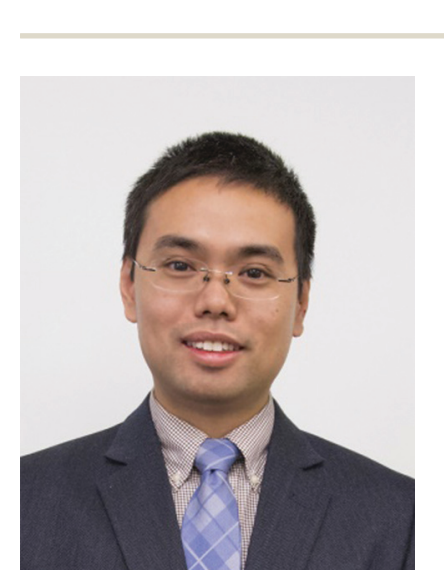
joined Central south University

Dr Yongbo Yuan is a Professor in Central South University. He received his BSc degree in physics from Sun Yat-sen (Zhongshan) University, China, in 2004 and his PhD degree in condensed matter physics from Sun Yat-sen (Zhongshan) University, China, in 2009. From 2009 to 2016 he conducted postdoctoral research in Prof. Jinsong Huang's group in University of Nebraska-Lincoln. After postdoctoral research, he as a Professor in the School of Physics and Electronics. His research interests includes: perovskite and polymer solar cells, organic thin film transistors and photodetectors. He won "Youth Thousand Talents Program" award of China in 2016, and has been identified in the 2018 Highly Cited Researchers list from Clarivate Analytics.

hybrid perovskites induce a large density of deep level traps and introduced the concept of passivation to the field of hybrid perovskites field. ${ }^{26,27}$ In semiconductor materials, non-radiative charge recombination generally occurs through deep level traps or Auger recombination when the concentration of charge carriers is high. ${ }^{23,24}$ Recent studies revealed Auger recombination is relatively weak in perovskite solar cells under 1 sun illumination. ${ }^{24,25}$ When imperfections create deep level traps, they will only trap either electrons or holes, which cannot escape with the assistance of thermal activation and will be annihilated with an oppositely charged carrier through non-radiative recombination, described by Shockley-Read-Hall (SRH) theory. ${ }^{28}$ SRH recombination through deep level traps has been determined to be the dominant pathway for the loss of charge carriers in perovskite materials. ${ }^{23,24}$ When imperfections create shallow level traps, the trapped charges can be re-emitted back into the CBM or VBM via phonon absorption. $^{29}$

There are four main types of imperfections in OIHP materials that have been identified as significant sources for non-radiative recombination: (1) some intrinsic point defects, ${ }^{18-21}$ (2) impurities, such as $\mathrm{Li}^{+}$from Spiro-OMETAD, Au from deposited electrodes, and many others from the raw materials, ${ }^{30,31}$ (3) two-dimensional extended defects, including grain boundaries and surface defects, ${ }^{32,33}$ (4) three-dimensional defects, like lead clusters. ${ }^{34,35}$ It should also be recognized that the strong non-radiative processes at the defective surface of films or crystals, although detrimental to solar energy conversion, can give rise to interesting applications of perovskite materials such as narrow-band photodetectors. $^{36}$ When considering the influence of various defects it is important to note that most point defects with low formation energies are reported to result in shallow level traps and thus have a negligible contribution to non-radiative recombination, possibly explaining the long diffusion lengths and the high defect tolerance of OIHP materials. ${ }^{17-21}$ However, due to the

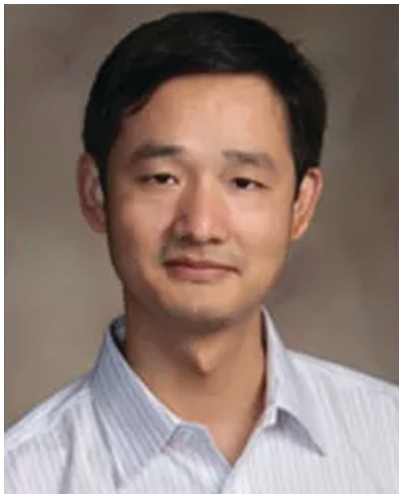

Jinsong Huang
Prof. Jinsong Huang is a Professor in the Department of Applied Physical Sciences at University of North Carolina at Chapel Hill. He received his $P h D$ degree in Material Science and Engineering from the University of California-Los Angeles in 2007. His current research interests include electronic materials and devices for applications in energy harvesting, sensing, and consumer electronics. 
ionic nature of OIHP materials and high ion migration speed, those charged point defects can migrate to interfaces under an electric field and influence the photovoltaic performance of PSCs. ${ }^{37-52}$ Therefore, in order to attain highly efficient and stable PSCs it is not only important to passivate defects responsible for deep level traps, but also effectively suppress migration of ions through the passivation of defect induced shallow level traps.

In this review, we describe the mechanisms of different methods for the passivation of imperfections in perovskite solar cells to reduce non-radiative charge recombination and suppress ion migration. We review the achievements that have been made to reduce the deep level traps by defect passivation for PSCs through coordinate bonding, ionic bonding, and conversion of extended imperfections to wide bandgap materials. The impacts of ion migration on band bending, interfacial reactions, and photo-induced phase segregation are summarized. The relation between imperfection passivation and device stability of PSCs is also discussed.

\section{Imperfections in perovskite and impact on perovskite solar cells}

The imperfections in OIHP films could influence the photovoltaic parameters of PSCs, such as open circuit voltage $\left(V_{\mathrm{OC}}\right)$, short-circuit current density $\left(J_{\mathrm{SC}}\right)$, fill factor $(\mathrm{FF})$, and PCE. During the operation of solar cells, incident light excites electrons from valence band into conduction band, splitting the quasi-Fermi levels of electrons and holes. The quasi-Fermi level splitting is controlled by the charge density at which the rate of charge recombination is equal to the rate of charge generation. The $V_{\mathrm{OC}}$ is derived from the splitting of hole and electron quasi-Fermi levels. When a non-radiative recombination process creates a drainage for the recombination of excess free charge carriers, it reduces the steady-state charge density, which reduces the splitting of quasi-Fermi levels and ultimately decreases the $V_{\text {OC }}$ of solar cells. The relationship between the $V_{\mathrm{OC}}$ and recombination can be described by the external luminescence quantum efficiency $\left(\eta_{\text {ext }}\right):^{24,53}$

$$
V_{\mathrm{OC}}=V_{\mathrm{OC}, \mathrm{rad}}+\frac{k T}{q} \ln \left(\eta_{\mathrm{ext}}\right)
$$

where $V_{\text {OC,rad }}$ is the radiative limit of the open circuit voltage when non-radiative recombination is fully suppressed. The dominant non-radiative recombination process occurs through $\mathrm{SRH}$ process due to the trapping effect of deep level defects when the free carrier density is relatively high. Thus it is important to de-activate and reduce the density of deep charge traps through electronic passivation. Moreover, the recombination at surface and grain boundaries also influence the charge extraction efficiency and could impact the $J_{\mathrm{SC}}$ and FF of PSCs. ${ }^{54}$ Defection passivation might also change the contact resistance at OIHP/charge transport layers interface and the addition of passivation agents in perovskite precursors may even change the film morphology with less pin-hole as shunt path, which leads to a changed FF in PSCs after defect passivation.

Fig. 1 illustrates the primary imperfections on the surface or grain boundaries of perovskite crystals that may cause deep level traps: undercoordinated halides ions, undercoordinated $\mathrm{Pb}^{2+}$ ions, lead clusters, and occasionally a few intrinsic point defects, such as $\mathrm{Pb}-\mathrm{I}$ antisite defects $\left(\mathrm{PbI}_{3}{ }^{-}\right)$, resulting from growth or processing conditions. Fig. 1 also illustrates some intrinsic point defects which are reported to form shallow level traps, such as I or MA vacancies in the bulk of the material. Due to the ionic nature of OIHP materials, the required passivation of charged defects in perovskite solar cells is different than covalent-bond based semiconductors like silicon. The charged

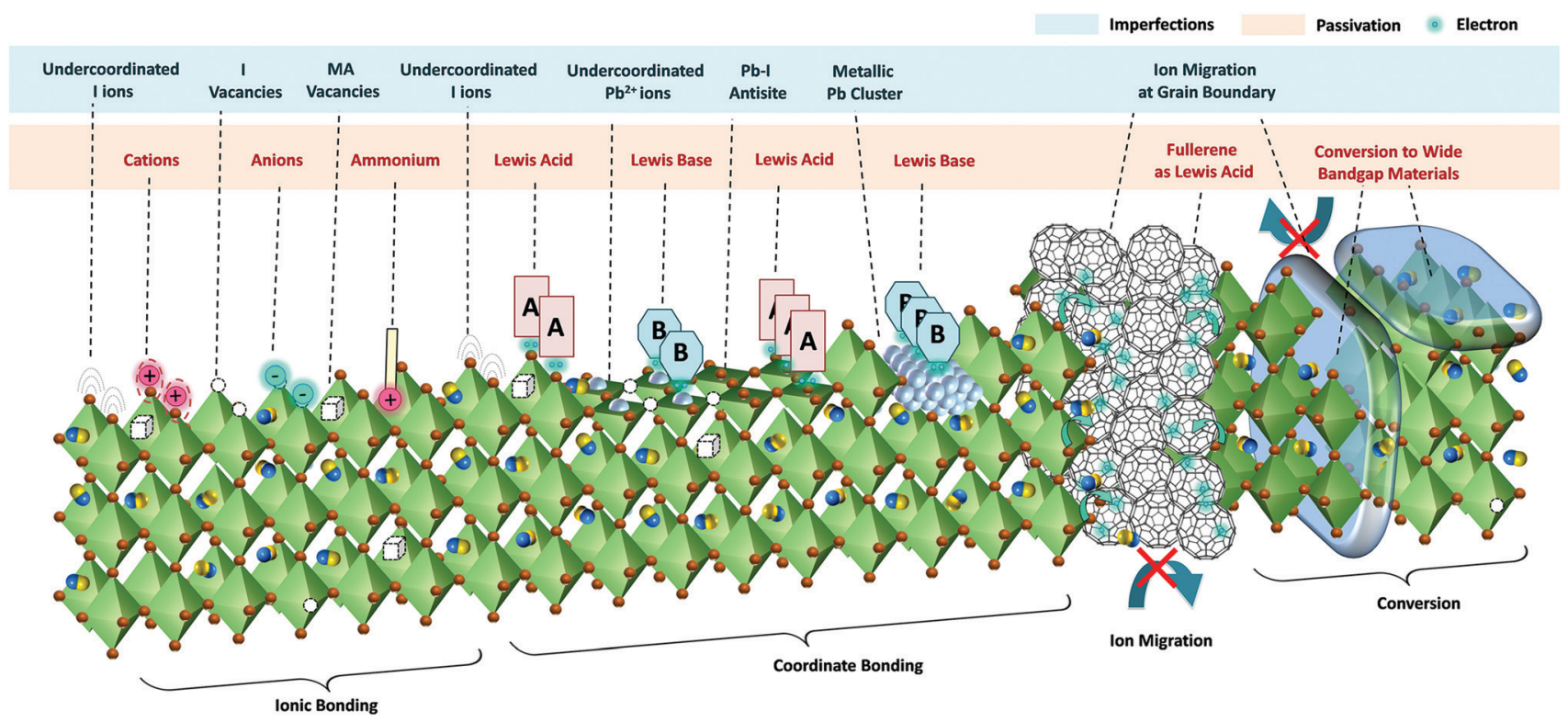

Fig. 1 Imperfections in OIHP film and their passivation by ionic bonding, coordinate bonding, and conversion to wide bandgap materials, and suppression of ion migration at extended defects. 
nature of defects in OIHP materials enables unique passivation methods like coordinate bonding (discussed in Section 3.1) or ionic bonding (discussed in Section 3.2) to neutralize and de-activate deep level traps resulting from charged defects. For example, the excess electrons of undercoordinated $\mathrm{I}^{-}$ions and $\mathrm{Pb}-\mathrm{I}$ antisite defects result in negatively charged defects that can then be passivated by a Lewis acid through coordinate bonding ${ }^{26,55,56}$ or by cations through ionic bonding. ${ }^{47,57-60}$ Additionally, similar to the passivation of dangling bonds at the surface of silicon materials by growth of silicon dioxide, silicon nitride, or aluminum oxide, ${ }^{56,61,62}$ converting the surface or grain boundaries of perovskites with a wide bandgap materials (discussed in Section 3.3) provides another approach to passivate the extended defects in OIHP materials.

Unlike deep level traps, shallow level traps originating from easily formed point defects are not detrimental to the photovoltaic performance of perovskite materials; ${ }^{17-19}$ however, the migration of such charged point defects under electric field could cause accumulation of charged ions at interfaces and result in unintentional doping effects. The migration or redistribution of these imperfections (e.g., vacancies, interstitials, or antisites) can result in local band bending, ${ }^{39,63}$ current density-voltage $(J-V)$ hysteresis, ${ }^{37,39-43}$ phase segregation, ${ }^{44-47}$ and degradation of perovskites or metal electrodes. ${ }^{48-52}$ This opens pathways for point defects associated with shallow level traps to influence the device performance in PSCs (discussed in Section 4). The migration of mobile imperfections in PSCs is most significant through grain boundaries of perovskite, ${ }^{64}$ increasing the need for extended defect passivation to hinder their migration pathways, as illustrated in Fig. 1 (discussed in Section 4.4).

It is noted that the dominant type of defects present in PSCs and their concentration is sensitive to the device fabrication processes and perovskite compositions. ${ }^{60,65-71}$ For example, perovskite precursors with non-stoichiometric $\mathrm{PbI}_{2}$ and MAI ratio could induce extended defects with different types of defects and also change the formation energy of point defects. ${ }^{18,60,67}$ Thermal annealing for a longer period of time or at higher temperatures can also result in the evaporation of organic cations and halides, leaving undercoordinated $\mathrm{Pb}^{2+}$ ions at surface or forming $\mathrm{PbI}_{2}{ }^{68-70}$ Another example is modulating perovskite composition via incorporating small isovalent ions to help suppress the formation of vacancies in OIHP films. ${ }^{71}$ Due to the variety of defect species and their densities, various passivation methods and passivating agents have been reported to be capable of effectively passivating perovskite materials.

Several techniques have been proposed to measure the density of states of traps, such as scanning tunneling microscopy (STM), ${ }^{72-74}$ thermal admittance spectroscopy (TAS), ${ }^{26}$ ultraviolet photoemission spectroscopy (UPS), ${ }^{32}$ thermally stimulated current (TSC), ${ }^{75}$ and deep level trapped defect analysis. ${ }^{76}$ However, it is still a challenge to pin-point the type of defects and then correlate that to the depth of the trap. Most of the known measurements, like TAS, UPS and TSC, are not specific to defect types. Although surface sensitive techniques such as STM have been used in attempt to study several surface defects of perovskite single crystals, ${ }^{72-74}$ the defect configuration is much more complicated in polycrystalline perovskite films formed by solution processing or evaporation. It turns out that the large roughness of most polycrystalline perovskite films poses a great challenge for successfully using STM to accurately study the defects in perovskite thin films. Nevertheless, the possible charge of defects present in perovskite, particularly at the surface, can be probed via the introduction of various molecules known to be capable of interactions with a given defect. The rational design of molecular structures for targeted passivation and analysis of their resulting efficacy can give insight to the configuration of defects present.

\section{Passivation of imperfections in perovskites}

Due to the ionic nature of OIHP materials, imperfections cause a non-stoichiometric balance of charges and result in either a positively or negatively charged defect site. Here we summarize the current approaches to de-active the defect induced deep traps by passivation through coordinate bonding, ionic bonding, and conversion to a more favorable material. Due to the presence of extended defects, such as grain boundaries and interfaces, and chemical instability of hybrid perovskites, there is likely a nonstoichiometric composition resulting in the presence of undercoordinated species. ${ }^{77}$ The evaporation of organic cations and halides can leave undercoordinated $\mathrm{Pb}^{2+}$ ions at the perovskite surface and grain boundaries. Undercoordinated $\mathrm{Pb}^{2+}$ has the ability to accept electrons making it characteristic of a Lewis acid, and then undercoordinated halides are characteristic of a Lewis base. These undercoordinated sites are then susceptible to reduction or oxidation (redox) reactions. The reduction of undercoordinated $\mathrm{Pb}^{2+}$ to metallic $\mathrm{Pb}\left(\mathrm{Pb}^{0}\right)$ has usually been detected on the perovskite surface by X-ray photoemission spectroscopy (XPS). ${ }^{35,78}$ The undercoordinated $\mathrm{I}^{-}$ions are susceptible to oxidization into volatile bimolecular iodine, $\mathrm{I}_{2}$, the release of which can cause irreversible decomposition of perovskites. In addition, the presence of $I_{2}$ in perovskite triggers chemical chain reactions and causes accelerated degradation of perovskite films. ${ }^{79}$ Most of $\mathrm{Pb}^{2+}$ and $\mathrm{X}^{-}$ions on the film surface are undercoordinated. These undercoordinated sites are detrimental to the performance of solar cell devices.

\subsection{Passivation by coordinate bonding}

Lewis acid-base chemistry has been used as an effective methodology for the passivation of undercoordinated sites (Fig. 1). The addition of Lewis acid or base additives can favorably coordinate to the undesired defects forming a Lewis adduct. Such adducts are linked by a dative bond (coordinate covalent bond), in which the two shared bonding electrons originate from the same atom, i.e., Lewis base. The passivation of harmful defects by Lewis acid-base chemistry has proven to be an effective technique for improving the performance and stability of halide perovskites.

3.1.1 Passivation by Lewis acid. Implementing a Lewis acid with the capability of accepting a pair of nonbonding electrons 
has the capability to passivate electron rich defects, which can also be considered as Lewis bases. The undercoordinated $\mathrm{I}^{-}$ and antisite $\mathrm{PbI}_{3}{ }^{-}$, may contribute to deep traps at the surface and grain boundaries of OIHP films, making their passivation crucial to enhanced solar cell performance. The addition of a Lewis acid can form a Lewis adduct with the undercoordinated halides and $\mathrm{PbI}_{3}{ }^{-}$, eliminating the corresponding trap and reducing undesired non-radiative recombination.

Fullerene $\left(\mathrm{C}_{60}\right)$ and its derivatives (PCBM, ICBA, etc.) have proven to be some of the most effective passivation agents employed for high-efficiency PSCs. ${ }^{26,80} \mathrm{C}_{60}$ and its derivatives proves to be a unique Lewis acid for its excellent electron accepting capability is not due to chemical reactivity, but is attributed to the strain resulting from its spherical shape. ${ }^{81}$
By accepting an electron the hybridization of $\mathrm{C}_{60}$ is modified to alter its shape and drastically reduce the intrinsic strain of the molecule, ${ }^{81}$ thus making it a good Lewis acid, more details mechanism can be found in ref. 81. Shao et al. were the first to discover the capability of PCBM, $\mathrm{C}_{60}$ functionalized with phenyl butyric acid methyl ester, as a highly effective passivation molecule for improving device performance by mitigating both shallow and deep traps (Fig. 2a and b). ${ }^{26}$ Passivation by a thin layer of PCBM could reduce the surface charge recombination and eliminate the photocurrent hysteresis of perovskite solar cells. ${ }^{26}$ Although the entire electronic and chemical mechanism for defect passivation by $\mathrm{C}_{60}$ and its derivatives has not been totally resolved, the chemical interaction of PCBM with OIHP materials was first reported by Xu et al. ${ }^{55}$ It was determined,
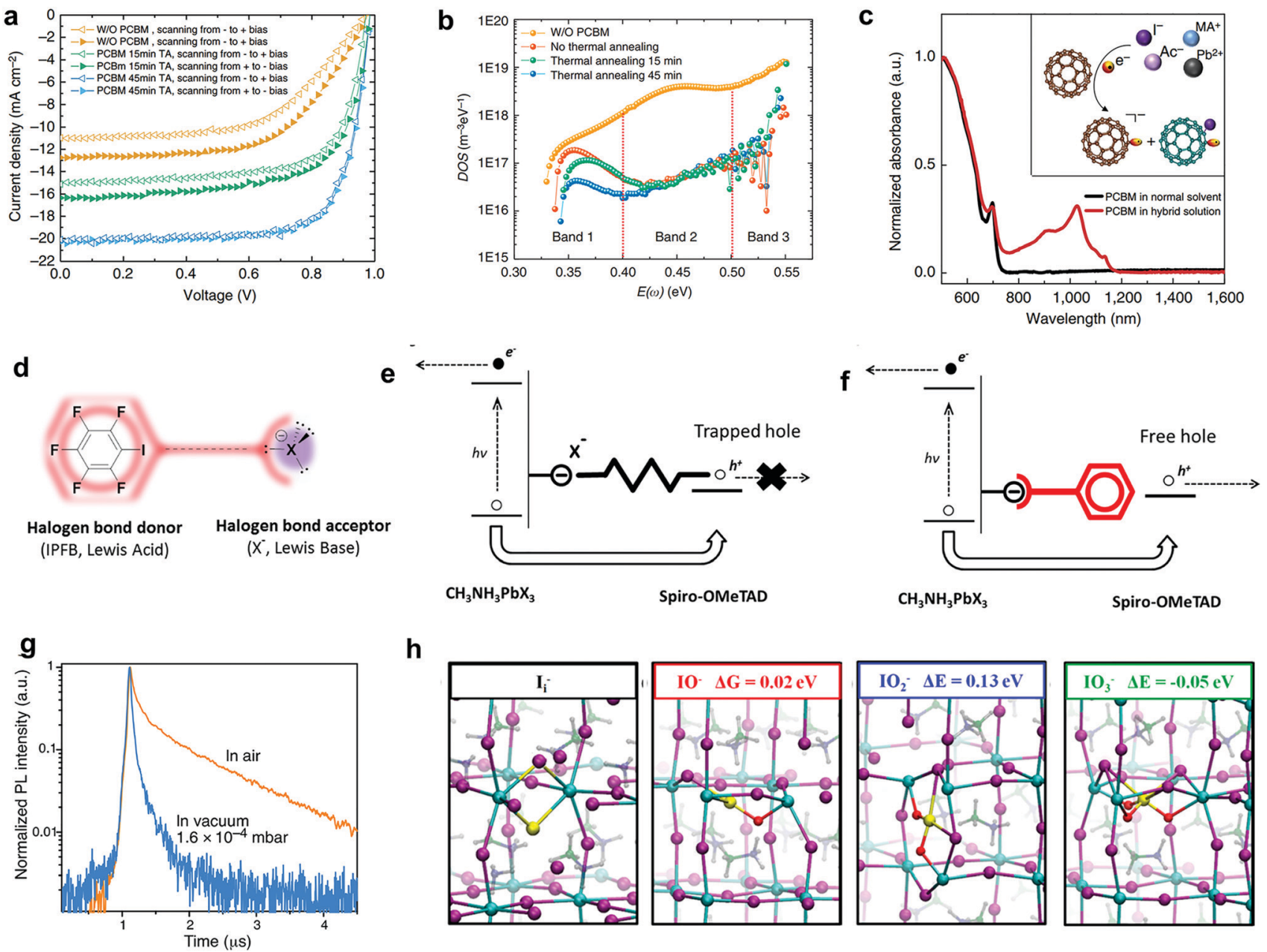

h
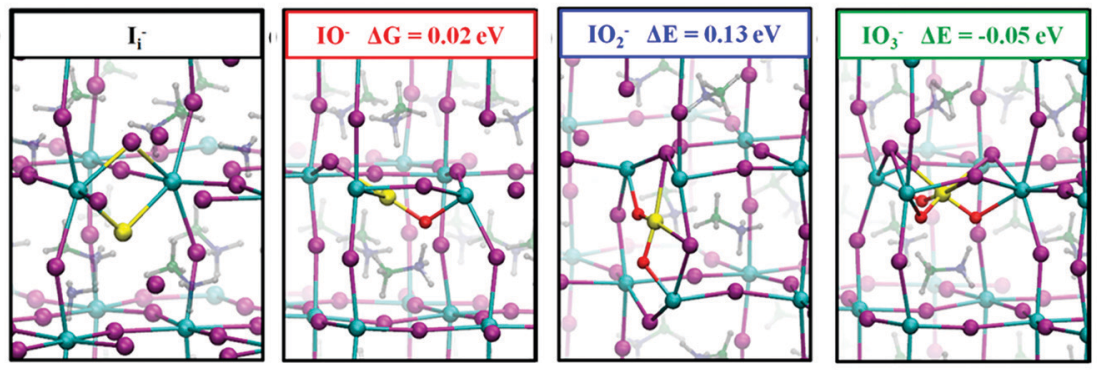

Fig. 2 Passivation by Lewis acids through coordinate bonding. (a) Photocurrents for devices without a PCBM layer, with PCBM layers thermally annealed for $15 \mathrm{~min}$ and $45 \mathrm{~min}$, respectively. (b) Trap density of states (tDOS) obtained by thermal admittance spectroscopy. Reproduced from ref. 26 with permission from Springer Nature, copyright 2014. (c) Ultraviolet-visible absorption spectroscopy of the hybrid solution shows the interaction between PCBM and perovskite ions. Reproduced from ref. 55 with permission from Springer Nature, copyright 2015. (d) Scheme of the halogen bond interaction between iodopentafluorobenzene and a generic halogen defect with $\mathrm{sp}^{3}$-hybridized valence electrons. Illustration of the charge trapping on the perovskite surface (e) without and (f) with iodopentafluorobenzene passivation. Reprinted with permission from ref. 27. Copyright 2014 American Chemical Society. (g) TRPL spectra of $\mathrm{MAPbBr}_{3}$ single crystal excited at a wavelength of $560 \mathrm{~nm}$ in air and vacuum. Reproduced from ref. 82 with permission from the AAAS, copyright 2016. (h) Structure of $\mathrm{I}_{\mathrm{i}}^{-}$and of its interaction products with $1 / 2 \mathrm{O}_{2}, \mathrm{O}_{2}$, and $3 / 2 \mathrm{O}_{2}$ along with the calculated energetics $(\Delta E, \mathrm{eV})$. Atom color code: yellow, $\mathrm{l}_{\mathrm{i}}$; purple, $\mathrm{I}$; red, $\mathrm{O}$; light blue, Pb. Methylammonium cations are shadowed in the background for clarity. Reprinted with permission from ref. 88. Copyright 2017 American Chemical Society. 
that in solution, PCBM reacted with $\mathrm{I}^{-}$to form a free radical (Fig. 2c). ${ }^{55}$ DFT calculations elucidated that when at a solid surface or grain boundary PCBM thermodynamically favors interaction with halide rich defects, while showing hybridization with the surface. ${ }^{55}$ This interaction is in good agreement with the mechanism for passivation by a Lewis acid.

Abate et al. have also demonstrated the passivation of undercoordinated halide ions by iodopentafluorobenzene via halogen bonding. ${ }^{27}$ They found that the highly electronegative fluorine atoms inductively withdraw electron density out of the aromatic ring of iodopentafluorobenzene, which reduces the electron density of the less electronegative iodine on the aromatic ring, -C-I (Fig. 2d). The electron deficient nature of this iodine makes it act as an electron acceptor, Lewis acid, for strong halogen bonding with an undercoordinated halide or $\mathrm{Pb}-\mathrm{X}$ antisite defect. The production of this $-\mathrm{C}-\mathrm{I} \cdots \mathrm{X}$ bond enhances the charge transfer at the perovskite/HTM interface, and increases the carrier lifetime by lowering the possibility of charge trapping at electron rich surface defects (Fig. 2e and f).

Besides the intentional passivation by chosen molecules, perovskite has been observed to be spontaneously passivated by moisture or oxygen in ambient air. ${ }^{82,83}$ There is no doubt that excess moisture and oxygen is detrimental to the perovskite structure, but a small amount has been reported to enhance the photovoltaic performance. ${ }^{84,85}$ The role of moisture in and at the surface of perovskite is complicated due to the difficulty in isolating the possible influential factors. One factor making understanding of this mechanism difficult is a water molecule's ability to be either an electron donor or acceptor depending on the chemical conditions. Some reports have claimed that water would contribute to the crystallization process of perovskite. ${ }^{86,87}$ When perovskite is exposed to oxygen and moisture, both the PL intensity and decay lifetime were obviously improved (Fig. 2g) because of the reduction of specific trap states. ${ }^{82,88}$ The twophoton excited fluorescence of $\mathrm{MAPbBr}_{3}$ single crystals exhibit the similar bulk recombination rate in both air and vacuum, indicating the passivation by water and oxygen is likely to occur at the surface of the crystal. ${ }^{82} \mathrm{D}$. Meggliolaro et al. proposed that oxygen is able to effectively deactivates deep hole traps associated with iodide interstitial sites $\left(\mathrm{I}_{\mathrm{i}}\right.$ ) by forming a moderately stable oxidized complex (Fig. 2h).$^{88,89}$ In this case, oxygen is a Lewis acid proving capable of reducing detrimental recombination events by eliminating deep traps in the bandgap of perovskite.

3.1.2 Passivation by Lewis base. Lewis bases are capable of donating a pair of nonbonding electrons in order to coordinate with, and passivate, undercoordinated $\mathrm{Pb}^{2+}$ or $\mathrm{Pb}$ clusters, forming a Lewis adduct. As shown in Fig. 3a, Noel et al. first proposed that the use of molecules containing a lone pair of electrons on either nitrogen (N) or sulphur (S) functionalities would work as a Lewis base to passivate defects in perovskite materials. ${ }^{90}$ Other functional groups with lone pair bearing atoms like oxygen $(\mathrm{O})$ or phosphorus $(\mathrm{P})$ also have Lewis base characteristic, passivation by these as well as $\mathrm{N}$ and $\mathrm{S}$ functionalities are summarized in Table 1. DFT calculations and Fourier Transform Infrared spectroscopy (FTIR) show strong evidence that these Lewis base functionalities have strong coordination with undercoordinated $\mathrm{Pb}^{2+}$, producing a Lewis adduct. ${ }^{97,101}$ The formation of such adducts can reduce the nonradiative charge recombination, improving lifetimes to enhance perovskite device performance and stability. Later, derivatives of the initially proposed pyridine and thiophene Lewis bases, were found to have a further improved passivation effect on perovskites. ${ }^{99,100}$

The use of oxygen containing alkylphosphine oxides as well as phosphorus of alkylphosphines have also been used as a Lewis bases in order to passivate the surface defects of perovskite. $^{92,98,102}$ deQuilettes et al. have investigated three Lewis bases, including trioctylphosphine oxide (TOPO), octadecanethiol and triphenylphosphine, and demonstrated TOPO treatment improved the PLQY of the perovskite thin-film from $\sim 3 \%$ to $35 \pm 1 \%$ and increasing the PL lifetime from $0.97 \mu$ s to as long as $8.82 \pm 0.03 \mu$ s at solar equivalent carrier densities. ${ }^{102}$ Braly et al. further characterized the PLQY and quasi-Fermilevel splitting of hybrid perovskite thin films with TOPO surface treatment reporting a remarkable increase of the internal PLQY from $9.4 \%$ to $91.9 \pm 2.7 \%$, under 1 sun equivalent photon flux. ${ }^{103}$ After the surface passivation by TOPO, a high quasiFermi-level splitting was measured to be $97.1 \pm 0.7 \%$ of the radiative limit, approaching that of the highest performing GaAs solar cells (Fig. 3c and d). ${ }^{103}$ These results clearly show the role of phosphorus and oxygen functionalities in reducing the non-radiative recombination, but more work is needed to explore for their applications in solar cells and other devices, as the long alkyl chains of the molecules discussed prove to be highly insulating.

Reduced graphene oxide (RGO) based materials containing oxygen based hydroxyl $(-\mathrm{OH})$ groups, although initially of interest as a hole transport layer (HTL) for pristine graphene's extremely high intrinsic charge carrier mobility of $\sim 200000 \mathrm{~cm}^{2} \mathrm{~V}^{-1} \mathrm{~s}^{-1,104,105}$ have proven to be more effective in improving grain growth and defect passivation. Using RGO, Li et al. observed the existence of $\mathrm{Pb}-\mathrm{O}$ bonds between $\mathrm{Pb}$ ions and hydroxyl groups $(-\mathrm{OH}) .{ }^{93}$ They speculated that the hydroxyl groups $(-\mathrm{OH})$ on RGO are capable of acting as a Lewis base to passivate undercoordinated lead defects. ${ }^{93}$ Hadadian et al. has reported that the nitrogen doped RGO (N-RGO) would further increase PL lifetimes relative to RGO, indicative of suppressed charge recombination due to improved passivation effect by the quaternary, pyrrolic, and pyridinic amines of $\mathrm{N}-\mathrm{RGO} .{ }^{96}$ A simple explanation for enhanced passivation by amines could be the lower electronegativity of nitrogen making it a better Lewis base relative to the oxygen of hydroxyl groups.

Other Lewis bases containing amines (such as $-\mathrm{NH}_{2}$ ) or nitrogen functionalities have also shown effective passivation of OIHP materials. ${ }^{94,97,106}$ Wang et al. also illustrated that spin-coating a diluted solution of phenylalkylamines, with the structure phenyl-R- $\mathrm{NH}_{2}$, on perovskite could effectively passivate the surface defects. ${ }^{94}$ Most impressively they reported an increase in PCE from 14.2 to $19.2 \%$ with a very small $V_{\text {OC }}$ deficit of $0.36 \mathrm{~V}$ for $\mathrm{FAPbI}_{3}$ based solar cells passivated by benzylamine. ${ }^{94}$ The benzylamine modified device showed longer carrier lifetime and improved diode characteristic, which should be due to the reduction of trap states related to 
a
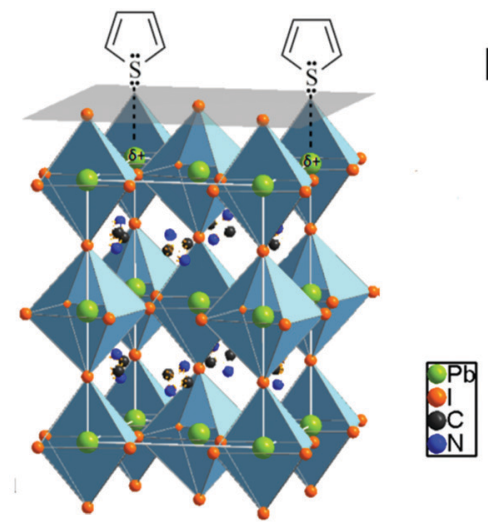

C

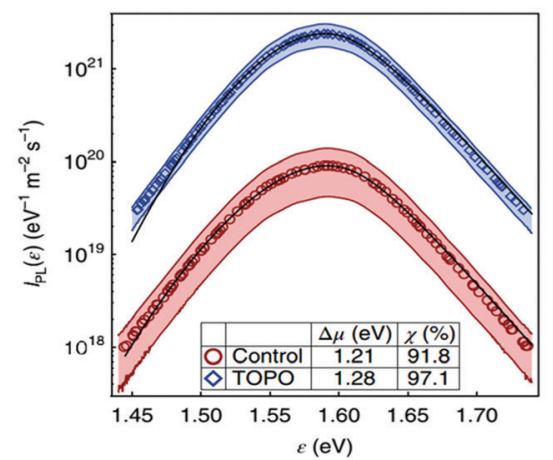

b
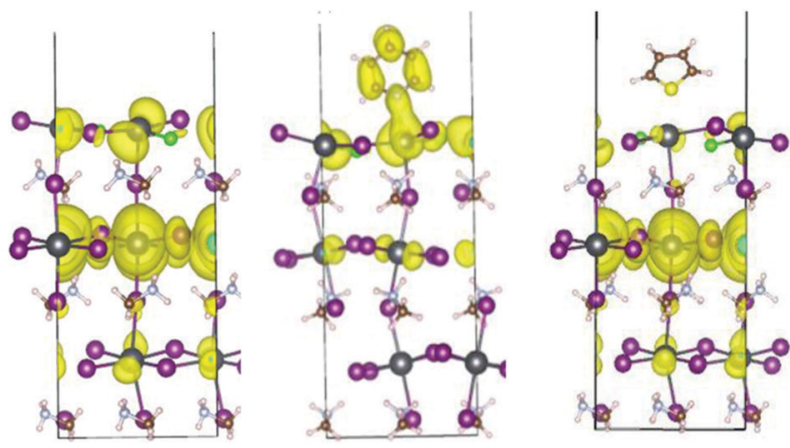

d
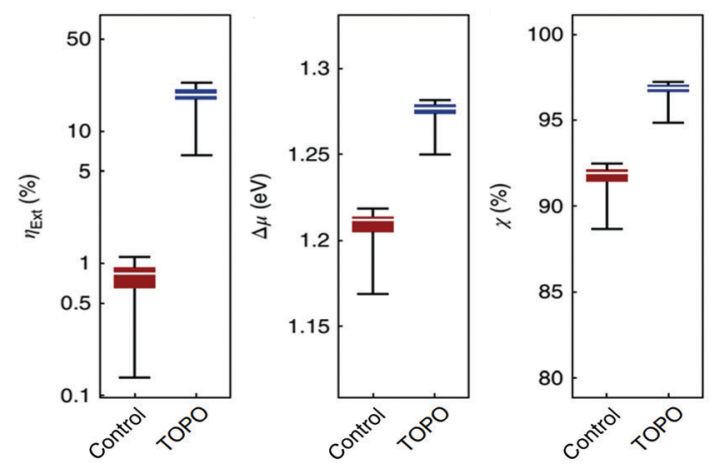

Fig. 3 Passivation by Lewis bases through coordinate bonding. (a) Passivation of under-coordinated $\mathrm{Pb}^{2+}$ by thiophene or pyridine on the surface of perovskite. Reprinted with permission from ref. 90. Copyright 2014 American Chemical Society. (b) LUMO charge density in (left) the bare Cl-doped $\mathrm{MAPb}_{3}(001)$ surface and the surface with adsorbed (middle) pyridine and (right) thiophene. Reprinted with permission from ref. 101. Copyright 2018 American Chemical Society. (c) Log-scale photoluminescence spectra with fits to the data (black lines) using the generalized Planck model. (d) Parameter spatial statistics (min, first quartile, median, third quartile, and max) of the control film and the TOPO-treated film. $H_{\text {ex }}, \Delta \mu$ and $\chi$ are internal PLQY, quasi-Fermi-level splitting and per cent of radiative limit quasi-Fermi level splitting, respectively. Reproduced from ref. 103 with permission from Springer Nature, copyright 2018.

surface defects. ${ }^{94}$ Notably, Zuo et al. demonstrated the use of poly(4-vynylpyridine) (PVP), a polymer functionalized with pyridine, as a passivation agent for $\mathrm{MAPbI}_{3}$ based PSCs, ${ }^{97}$ and the Lewis base pyridine functionalities are able to passivate the undercoordinated $\mathrm{Pb}^{2+}$ in order to achieve a PCE of $20.23 \%$, compared to $18.05 \%$ of the untreated device. ${ }^{97}$

Furthermore, Lin et al. employed $\pi$-conjugated small molecules, indacenodithiophene end-capped with 1.1-dicyanomethylene3-indanone, as an electron donor to passivate the Lewis acid traps of hybrid perovskites. ${ }^{95}$ Such molecules combine the advantages of electrical conductive organic $\pi$-conjugated structures like graphene and the reduction of specific defects by the coordination with typical Lewis base functional groups (e.g., thiocyanato, sulfhydryl, amine, etc.). ${ }^{95}$

\subsection{Passivation by ionic bonding}

Ionic bonding involves the complete transfer of one or more valence electrons from one atom to another to afford an electrostatic interaction between two ions of complementary charge, i.e., a positively charged cation and a negatively charged anion. Due to the charged nature of defects in perovskite, ionic bonding can be used as another unique passivation technique. The selective introduction of ions with charge complementary to a given defect can bond with the defect and effectively annihilate the corresponding trap states. Passivation by ionic bonding has recently gained significant interest for its proven ability to improve the stability and efficiency of PSCs.

3.2.1 Passivation by cations. Cationic species including metal ions and organic molecules have been studied for their passivating effects of anionic defects, such as undercoordinated $\mathrm{I}^{-}$, antisite $\mathrm{PbI}_{3}{ }^{-}$, and $\mathrm{MA}^{+}$vacancies, in halide perovskites. Through ionic bonding and other electrostatic interactions with the negatively charged defects of perovskite materials, metal ions and organic cations have proven to be effective passivating agents. The capability for metal ions to have a passivation effect was first reported when $\mathrm{Bi}$ et al. found that sodium ions, $\mathrm{Na}^{+}$, present on substrates were capable of diffusing into grain boundaries over time. ${ }^{57}$ These $\mathrm{Na}^{+}$rich grain boundaries resulted in a lower defect density and improved PL lifetimes, suggestive of the efficient passivation of defective grain boundaries. ${ }^{57} \mathrm{Bi}$ et al. proposed that $\mathrm{Na}^{+}$could potentially passivate negatively charged defects, either by being adsorbed at MA vacancies of the grain boundaries, due to its similar size and isovalent charge relative to $\mathrm{MA}^{+}$, or form an ionic bond with undercoordinated halides. ${ }^{57}$ Abdi-Jalebi et al. later reported that if potassium iodide (KI) was added to a $\mathrm{Cs}_{0.06} \mathrm{FA}_{0.79}$ $\mathrm{MA}_{0.15} \mathrm{~Pb}\left(\mathrm{I}_{0.85} \mathrm{Br}_{0.15}\right)_{3}$ perovskite precursor, the potassium ions $\left(\mathrm{K}^{+}\right)$ 


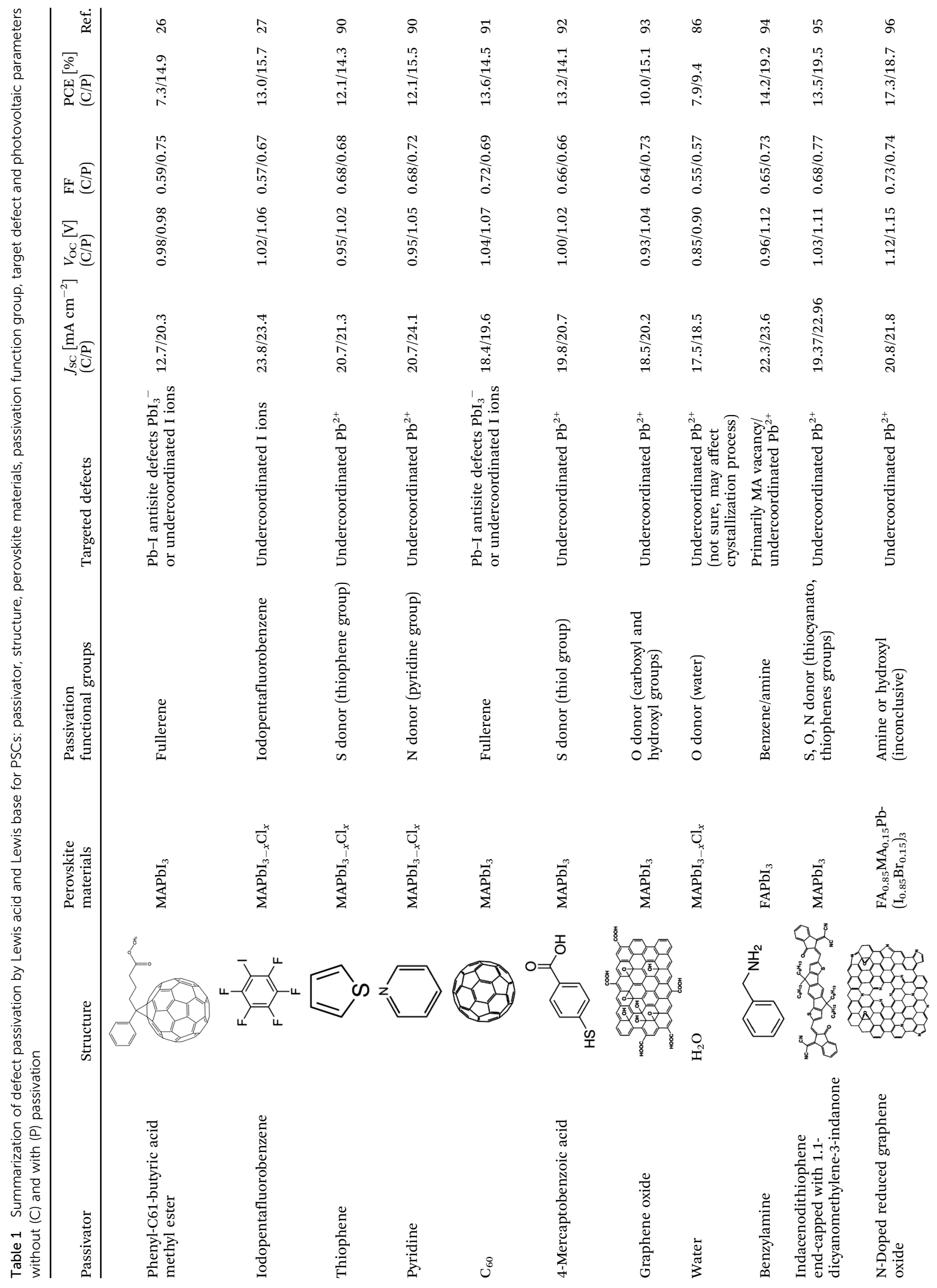


could accumulate at the surface and grain boundaries to passivate those extended defects to produce perovskite films with internal PLQYs > 95\% (Fig. 4a). ${ }^{47}$ This improvement in PLQY is attributed $\mathrm{K}^{+}$passivating the undercoordinated halides, both undercoordinated $\mathrm{Br}^{-}$and $\mathrm{I}^{-}$, at the surface and grain boundaries via ionic interactions (Fig. 4b). Through bonding with the halides, $\mathrm{K}^{+}$is also effective in suppressing ion migration (discussed with more details in Section 4.4). The ionic interaction between $\mathrm{K}^{+}$and halides at grain boundaries that has been reported thus far is supported by the particularly interesting work of Kubicki et al. using solid state nuclear-magnetic resonance to confirm the presence of KI while no change in the local environments of $\mathrm{N}$, and $\mathrm{C}$ atoms was observed. ${ }^{107}$ However, it is worth noting that calculations by Son et al. show that small alkali metal ions like $\mathrm{K}^{+}$, with radii of $1.38 \AA$, could be energetically favorable to occupy the interstitial sites within the perovskite lattice. ${ }^{108}$ These filled interstitial sites could prevent the formation of iodide Frenkel defects and hinder ion migration and result in a similar passivation effect to ionic bonding at the surface and grain boundaries. It is evident that $\mathrm{K}^{+}$and other metal ions, such as $\mathrm{Cu}^{+}, \mathrm{Ag}^{+}$, and $\mathrm{Rb}^{+}$, are capable of effectively passivating, but the true mechanism has yet to be fully divulged due to the conflicting reported position of metal ions within lattice or at the surface and grain boundaries. ${ }^{107-113}$ Additional research on a mechanism of metal ions would be greatly beneficial to the field moving forward.

Beyond metal ions, significant research has also been done looking at organic cationic passivating agents, such as those with ammonium $\left(-\mathrm{NH}_{3}{ }^{+}\right)$functionalities. Unlike amines, like $-\mathrm{NH}_{2}$, with a lone pair of electrons capable of coordinating to positively charged Lewis acid defects via coordinate bonding, the $-\mathrm{NH}_{3}{ }^{+}$cations passivate negatively charged defects through electrostatic interactions including ionic bonding and hydrogen bonding. A number of small organic molecules owning ammonium functionalities have been explored for their ability to enhance device performance and stability (Table 2), such as butylammonium $\left(\mathrm{BA}^{+}\right)$, octylammonium $\left(\mathrm{OA}^{+}\right)$, phenylethylammonium $\left(\mathrm{PEA}^{+}\right)$, and diammonium derivatives. ${ }^{58,114-119}$ Most notably, $\mathrm{BA}^{+}$and $\mathrm{PEA}^{+}$have been studied extensively for their ability to suppress trap induced recombination. It is important to note that $\mathrm{BA}^{+}$, $\mathrm{PEA}^{+}$, and other alkylammonium molecules have been reported to be capable of inducing a conversion of perovskite from a 3D to a layered phase, ${ }^{116-118}$ to be discussed with further detail in Section 3.3.2. Although the conversion results in a $3 \mathrm{D} /$ /ayered interface, the original defects present at the surface and grain boundaries can be eliminated due to lattice matching of the two phases, ${ }^{117}$ potentially passivating the perovskite surface and grain boundaries. The resulting band alignment of the $3 \mathrm{D} /$ layered interface has also proven to be beneficial and will be further discussed in Section 3.3.2. This makes it difficult to clearly deconvolute the contribution to passivation by solely ionic bonding of ammonium derivatives at the surface and grain boundaries.

It is often generalized that most alkylammonium derivatives with a carbon chain too long or too large to incorporate into the A site of the perovskite lattice will induce the formation of a lower dimensional perovskite material; ${ }^{120,121}$ however, improvement 

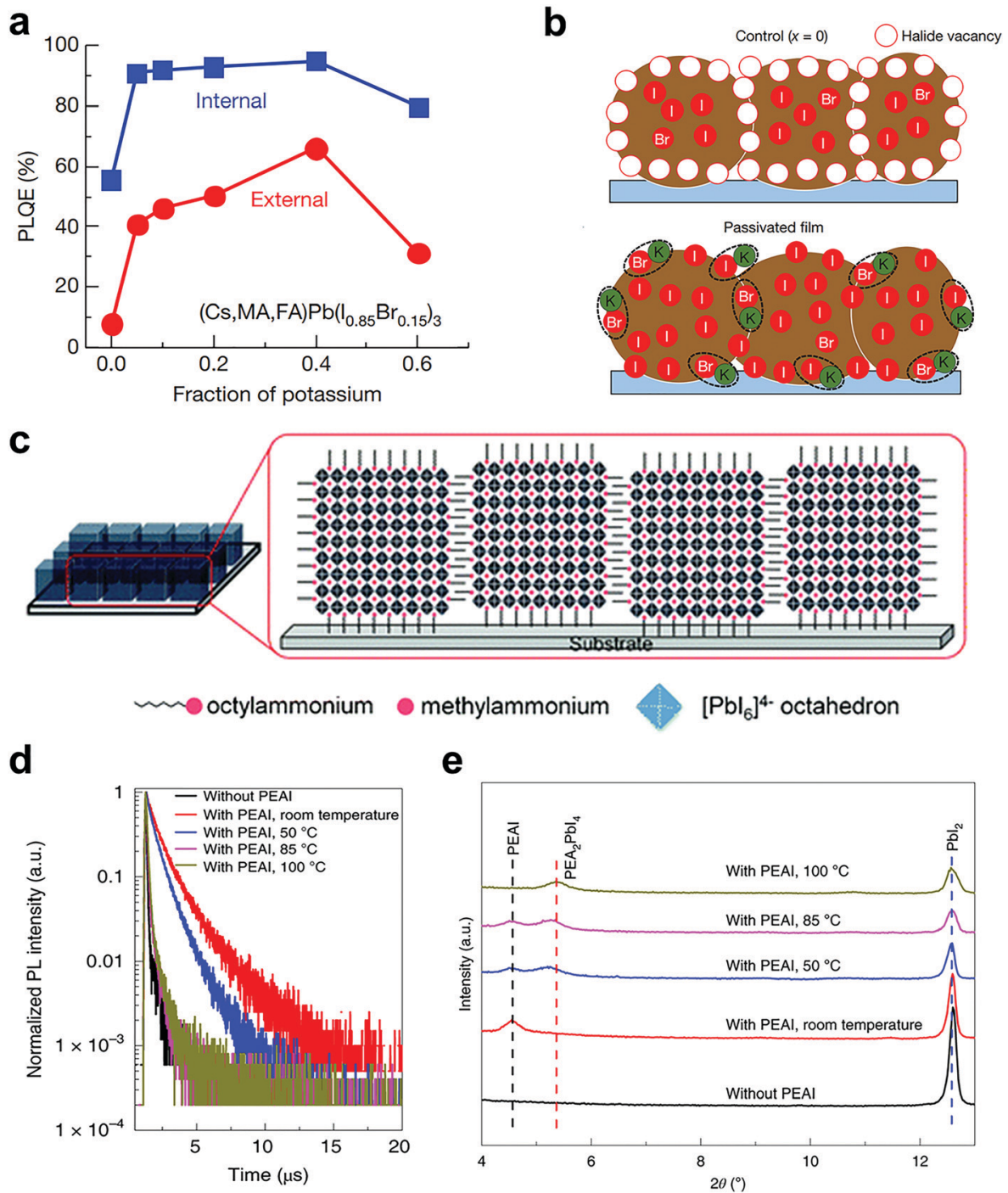

methylammonium

$\left[\mathrm{Pbl}_{6}\right]^{4 \cdot}$ octahedron

e

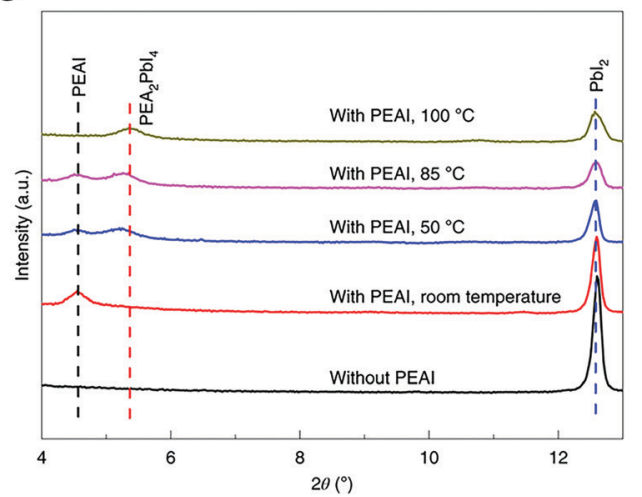

Fig. 4 Passivation by cations through ionic bonding. (a) PLQE of KI passivated perovskite thin films with increasing fraction of potassium. (b) Schematic of a cross-section of a film showing halide-vacancy management in cases of excess halide, in which the surplus halide is immobilized through complexing with potassium into benign compounds at the grain boundaries and surfaces. Reproduced from ref. 47 with permission from Springer Nature, copyright 2018. (c) Schematic illustration of PEA-modified perovskite films. Reproduced from ref. 58 with permission from Royal Society of Chemistry, copyright 2018. (d) Time-resolved PL and (e) XRD of the $\mathrm{FA}_{0.92} \mathrm{MA}_{0.08} \mathrm{Pbl}{ }_{3}$ films with PEAl treatment under different conditions. Reproduced from ref. 122 with permission from Springer Nature, copyright 2019.

in recombination lifetimes and $V_{\mathrm{OC}}$ without the observed formation of a layered phase was first reported by Zhao et al. using octane-1,8diammonium, suggesting passivation by ammonium functionalities via ionic or hydrogen bonding at surface and grain boundaries of perovskite. ${ }^{114}$ A similar passivation mechanism has been demonstrated by Jung et al. utilizing $\mathrm{OA}^{+},{ }^{58}$ showing no layered phase and reporting recombination lifetimes five times longer than control samples and improvement in PCE from $18.4 \%$ to $20.6 \%$ (Fig. $4 \mathrm{c}$ and Table 2) ${ }^{58}$ The $\mathrm{OA}^{+}$decorated surface also proved to improve the thermal and moisture stability of the OIHP material, ${ }^{58}$ additional discussion on the influence of passivating agents on stability will be covered in Section 5.

Although $\mathrm{PEA}^{+}$has been reported as one of the most effective additives for passivation via conversion to a layered phase, ${ }^{118}$ recent work by Jiang et al. has demonstrated excellent surface passivation with no evidence of a layered phase forming (Fig. 4d and e). ${ }^{122}$ They utilized PEAI $\left(\mathrm{PEA}^{+} \mathrm{I}^{-}\right.$) surface treatment on $\mathrm{FA}_{0.92} \mathrm{MA}_{0.08} \mathrm{PbI}_{3}$ without thermal annealing to provide PSCs reaching a certified efficiency of $23.32 \% .^{122}$ The 3D-to-layered perovskite conversion by $\mathrm{PEA}^{+}$likely still has a low energetic barrier that cannot be overcome without thermal annealing. XRD measurements also show the formation of layered perovskite $\left(\mathrm{PEA}_{2} \mathrm{PbI}_{4}\right)$ at higher temperatures, ${ }^{122}$ presumably indicating the activation barrier for conversion can be overcome by thermal energy (Fig. 4e). The use of cationic atoms and molecules have shown great potential for passivation, but it is evident that greater understanding on the influence of molecular design and processing is necessary to further improve their efficacy.

3.2.2 Passivation by anions. Despite significant developments in the use of cationic passivating agents, not much work 


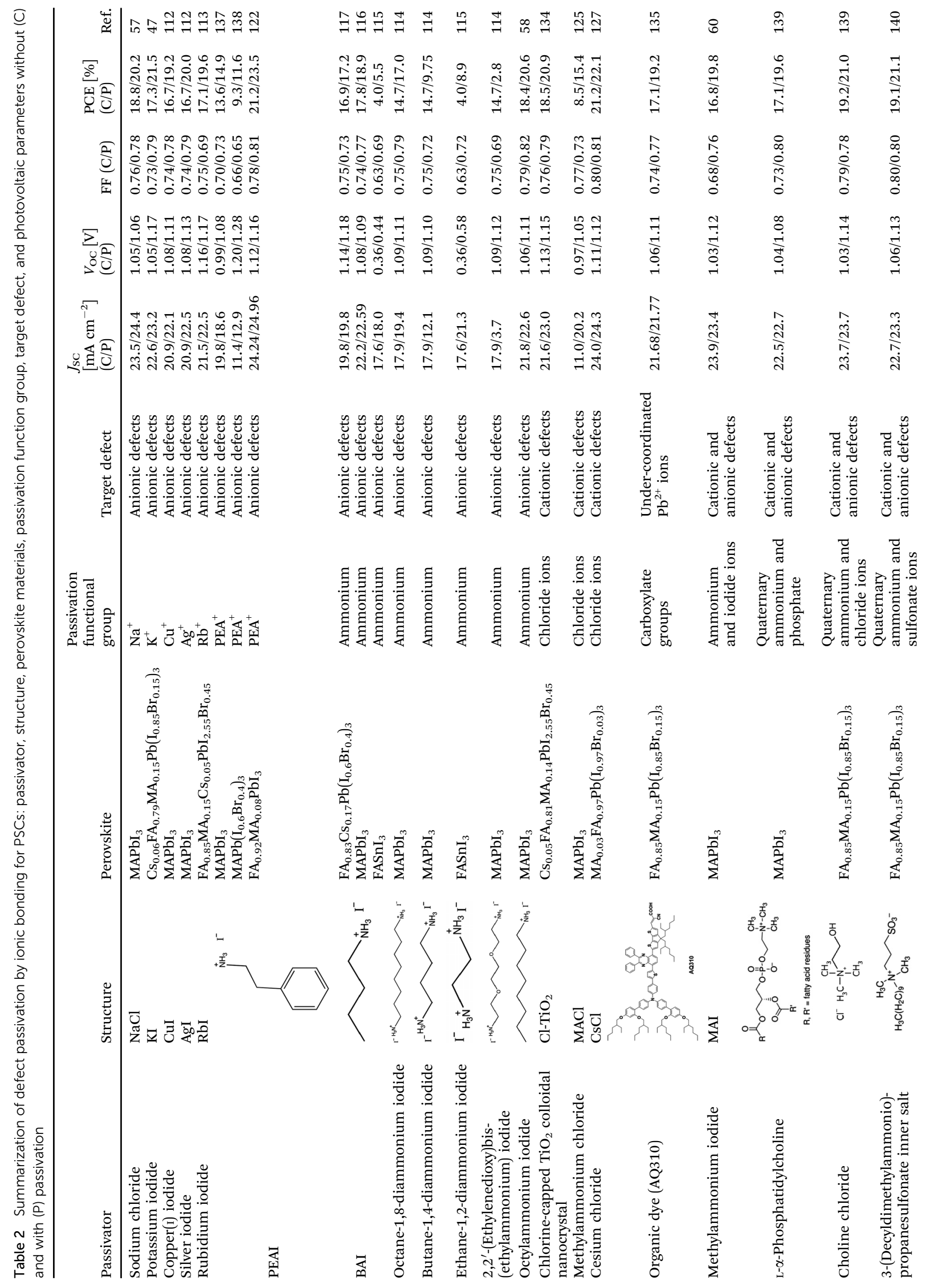


has been done exploring passivation with anionic alternatives, such as such as undercoordinated $\mathrm{Pb}^{2+}$ ions and halide vacancies. One reported anion for defect passivation of OIHP materials is chloride ion $\left(\mathrm{Cl}^{-}\right)$. Chloride ions have been introduced in various different forms, such as $\mathrm{PbCl}_{2}, \mathrm{NH}_{4} \mathrm{Cl}, \mathrm{MACl}, \mathrm{FACl}$, and CsCl. ${ }^{123-128}$ The addition of $\mathrm{Cl}^{-}$has been reported to have two different functions. ${ }^{129}$ The addition of $\mathrm{Cl}^{-}$could alter the electronic properties of films by doping perovskite or passivating defect induced traps at interfaces and/or grain boundaries; additionally, $\mathrm{Cl}^{-}$has shown influence over morphology by altering crystallization to provide large grain and smooth structural discontinuities at grain boundaries. ${ }^{129}$ Even though chloride ions have been demonstrated to easily leave perovskite films during thermal annealing, ${ }^{130}$ it is reported that there are still some residual $\mathrm{Cl}^{-}$ions remaining in thin films of $\mathrm{MAPbI}_{3} \cdot{ }^{129,131}$ In order to decouple the electronic effect and morphological influence of chloride ions, Chen et al. fabricated the perovskite films with similar polycrystalline texture and comparable grain sizes for samples with and without added $\mathrm{Cl}^{-} .{ }^{132}$ The transient photovoltage decay showed that devices with added $\mathrm{Cl}^{-}$resulted in charge lifetimes twice as long as control devices. ${ }^{132}$ The defect activation energy for the traps determined by capacitance-voltage measurements shifted from 74.4 to $21.6 \mathrm{meV}$ in the presence of $\mathrm{Cl}^{-},{ }^{132}$ which indicates a shift in the trap states to a shallower level. Therefore, the incorporated $\mathrm{Cl}^{-}$could improve the photovoltaic performance of PSCs by improving electronic properties. Simulation by Nan et al. shows that the deep trap states created by lead and iodine vacancies can be healed by introducing a tiny amount of $\mathrm{Cl}^{-} .{ }^{133}$ Tan et al. have also shown that the $\mathrm{Cl}$ of Cl-capped $\mathrm{TiO}_{2}$ can acting as an effective passivation agent at the interface of perovskite as the formation energy of $\mathrm{Pb}-\mathrm{Cl}$ antisite defects is much greater than that of a $\mathrm{Pb}-\mathrm{I}$ antisite, which indicates the antisite defects are suppressed in the presence of interfacial $\mathrm{Cl}$ atoms. ${ }^{134}$ In addition to being more difficult to form, $\mathrm{Pb}-\mathrm{Cl}$ antisite defects are calculated to result in shallower level traps that are delocalized over a larger area, making them less detrimental to device performance. ${ }^{134}$

Introduction of excess iodide, such as KI and MAI, has been reported could compensate the halide vacancies at the grain boundaries, and thus passivating the nonradiative recombination pathways. ${ }^{4760}$ Moreover, the use of anionic carboxylate groups $\left(-\mathrm{COO}^{-}\right)$, have been suspected to passivate positively charged defects in perovskite films through ionic bonding. ${ }^{135,136}$ However, it needs to be highlighted that carboxyl groups $(-\mathrm{COOH})$ and $-\mathrm{COO}^{-}$should not passivate through the same mechanism or address the same defects, which has yet to be clearly differentiated in reported works. ${ }^{135,136}$ Due to carboxyl groups being weak acids, whether it is protonated as $-\mathrm{COOH}$ or deprotonated to $-\mathrm{COO}^{-}$is dependent upon the dissociation constant, and resulting $\mathrm{p} K_{\mathrm{a}}$, of the carboxyl group and $\mathrm{pH}$ of the solution. Unlike $-\mathrm{COO}^{-}$ passivating positively charged defects through ionic interactions, - $\mathrm{COOH}$ should be capable of passivating negatively charged undercoordinated halides via hydrogen bonding.

3.2.3 Passivation by zwitterions. Zwitterions possess both positively and negatively charged functional groups, a unique property that has proved beneficial in improving photovoltaic performance of PSCs. ${ }^{139-141}$ One of the most promising applications of zwitterions is the capability to simultaneously passivate negatively and positively charged ionic defects using both mechanisms discussed so far. Zheng et al. first reported choline zwitterions, such as L- $\alpha$-phosphatidylcholine, choline chloride, choline iodide, can passivate both types of charged defects in perovskites (Fig. 5a). ${ }^{139} \mathrm{~L}-\alpha$-Phosphatidylcholine has the positive charged quaternary ammonium group $\left(-\mathrm{N}\left(\mathrm{CH}_{3}\right)_{3}{ }^{+}\right)$ and negative charged phosphate $\left(-\mathrm{PO}_{4}{ }^{-}\right)$; choline chloride and choline iodide contain the positively charged quaternary ammonium and negatively charged halides. The passivation is achieved by spin coating a thin layer of those choline zwitterions on perovskite films. $\mathrm{L}-\alpha$-Phosphatidylcholine passivation demonstrated an increase of $V_{\mathrm{OC}}$ from $1.04 \mathrm{~V}$ to $1.08 \mathrm{~V}$ and an increase of PCE from $17.1 \%$ to $19.6 \%$ compared to control device with PCBM, and choline chloride has exhibited a small $V_{\text {OC }}$ deficit of only $0.39 \mathrm{~V}$ and a high efficiency of $21.0 \% .^{139}$ Theoretical analysis suggested that those zwitterions can passivate two typical deep surface defects, anionic $\mathrm{Pb}-\mathrm{I}$ antisites and cationic $\mathrm{Pb}$ clusters. ${ }^{139}$ It was found that in the case of $\mathrm{Pb}-\mathrm{I}$ antisite defects, that choline can transfer about 0.8 electrons to the perovskite surface to effectively reduce the electronic trap states due to the defect. ${ }^{139}$ The excess halide ions introduced via the choline salt can also prevent the trapping of charges by forming new hybridized states which bridge the trap states with the states of the conduction band edge. ${ }^{139}$ Later, Zheng et al. added 3-(decyldimethylammonio)-propane-sulfonate inner (DPSI) salt, a sulfonic zwitterion containing a cationic quaternary ammonium group and an anionic sulfonic group $\left(-\mathrm{SO}_{3}{ }^{-}\right)$, into the perovskite precursor to provided effective passivation and boost the PCE of planar perovskite devices from 19.1\% to $21.1 \%$. $^{140}$

Similar in concept to zwitterions, other molecules have been designed with various functional groups capable of passivating multiple species of defects through a combination of mechanisms reviewed so far. Yang et al. proposed multifunctional passivation by D-4-tert-butyl-phenylalanine (D4TBP), a molecule with a carboxyl, amine and tert-butyl-phenyl functionalities which are capable of passivating multiple types of defects. ${ }^{136}$ This strategy yielded an open-circuit voltage of $1.23 \mathrm{~V}$ at a bandgap of $1.57 \mathrm{eV}$ with the loss in potential of only $0.34 \mathrm{~V}^{136}$ Interestingly, although conjugated rings have no charge, Yang et al. suggested the potential for phenyl groups to reduce defects associated with $\mathrm{I}_{2}$ by demonstrating formation of charge transfer complexes between the conjugated ring and $\mathrm{I}_{2},{ }^{136}$ while earlier research by Wei et al. and others have demonstrated the ability for the conjugated rings of rubrene to interact with $\mathrm{MA}^{+}$via electrostatic $\pi$-cation interactions. $^{142,143}$ The ability for these neutral conjugated organic molecules to interact and potentially passivate various defect species makes them a unique multifunctional passivating agent deserving of further studies to determine their capabilities. Overall, more work is expected to further characterize the structure-function relationship of all passivating agents discussed thus far. 
a

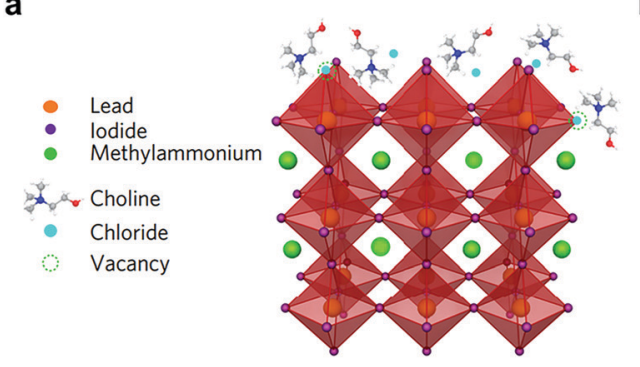

b
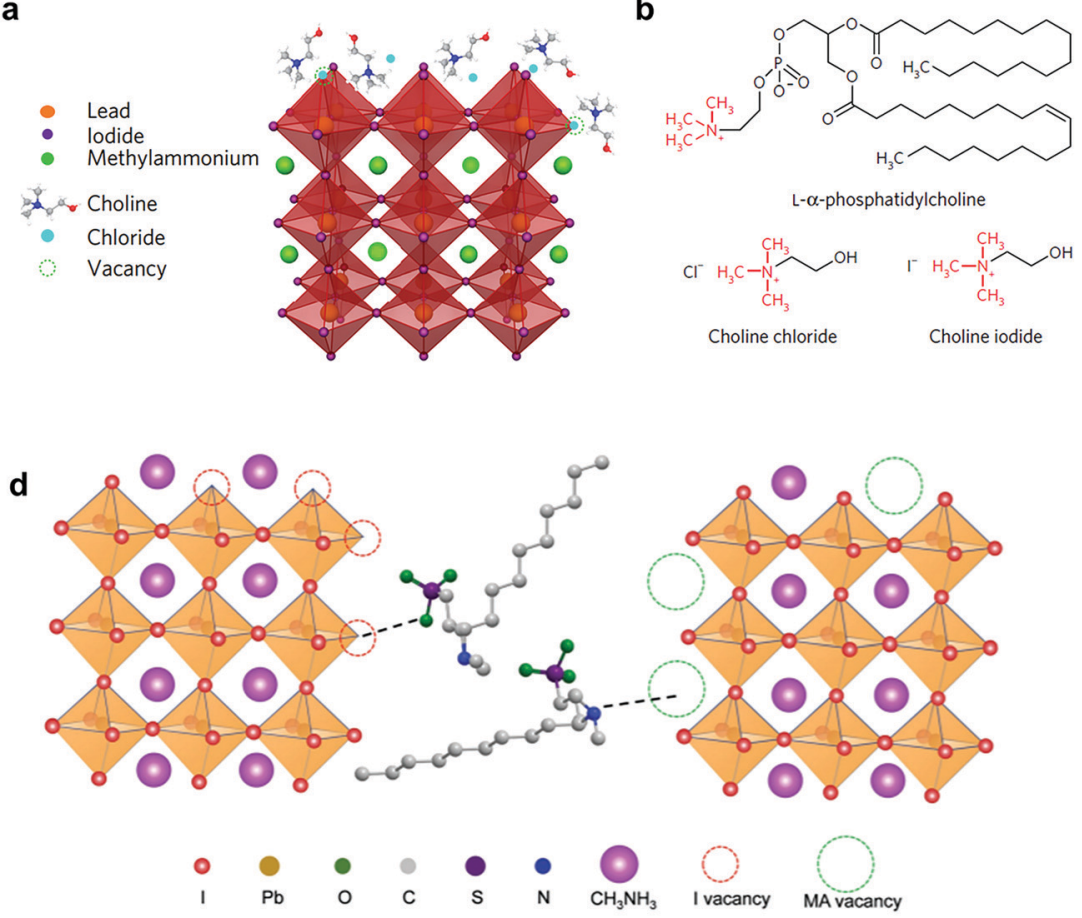

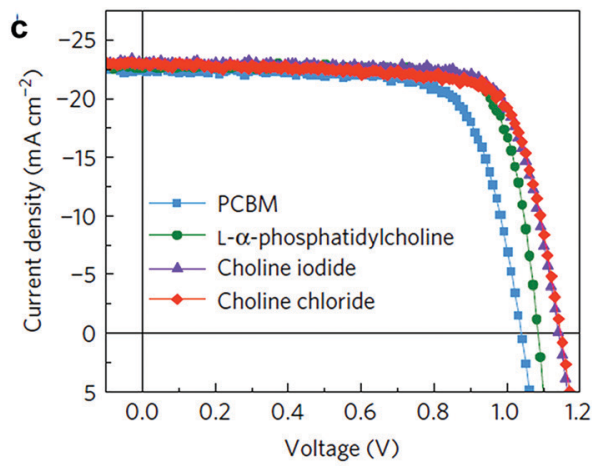

e

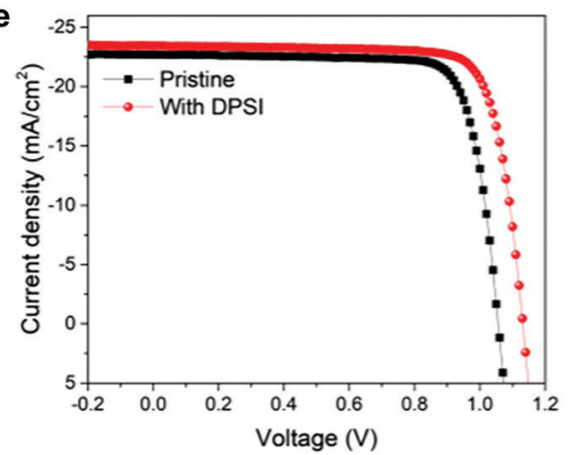

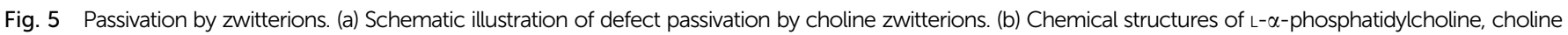

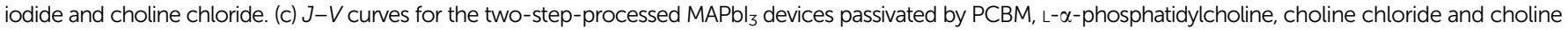

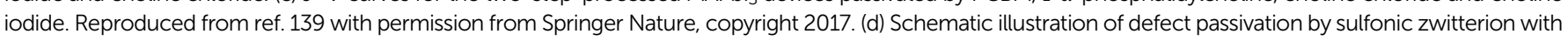

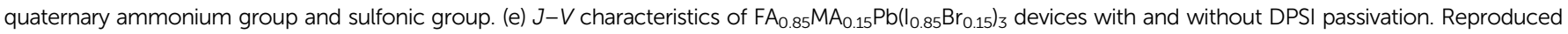
from ref. 140 with permission from John Wiley and Sons, copyright 2016.

\subsection{Conversion of extended imperfections to wide bandgap materials}

The ionic nature of defect rich surfaces and grain boundaries of perovskite have allowed for the use of Lewis acid-base chemistry and ionic bonding to passivate the extended imperfections; however, it is also possible to chemically convert the surfaces and grain boundaries to a material capable of either eliminating those extended defects or creating an interface with favorable band alignment. For commercialized silicon solar cells, conversion of surface dangling bonds to wide bandgap $\mathrm{SiO}_{2}$ and $\mathrm{SiN}_{x}$ is a well-established approach to passivate the surface defects. ${ }^{56,61,62}$ The choice option thus far has been the strategic conversion of the perovskite surface and grain boundaries to a wide bandgap semiconductor, ${ }^{68,116-119}$ which not only could eliminate the deep level traps at surface and grain boundaries but also form a type-I heterojunction to further hinder non-radiative recombination. For the type-I band alignment, the conduction band minimum (CBM) of the wide bandgap semiconductor lies above the CBM of the active perovskite, and its valence band maximum (VBM) is lower than the active perovskite's VBM. This type-I band alignment creates an electronic barrier to hinder the charge transfer between perovskite grains and also between perovskite and charge transport layers. Therefore, when charges reach the wide bandgap wrapping layer, instead of becoming trapped and recombining at the grain boundaries or interface, they will be reflected back into the bulk. Although this conversion could effectively suppress trap induced recombination at the extended defects, it is also critical to control the thickness of the wide bandgap materials at the perovskite/CTL interface for charges rely on tunneling to transfer through the wide bandgap material to be extracted by charge transport layer.

3.3.1 Conversion of extended imperfection by controlled degradation. The first prototype of type-I alignment in perovskite solar cells to reduce carrier recombination was reported by Chen et al. ${ }^{68}$ They controlled the annealing time of $\mathrm{MAPbI}_{3}$ film to tune the amount of $\mathrm{PbI}_{2}$ wrapping the perovskite at grain boundaries and the perovskite/CTL interface. $\mathrm{PbI}_{2}$ is formed due to release of MAI during the decomposition of $\mathrm{MAPbI}_{3}$. Annealing at $150{ }^{\circ} \mathrm{C}$ for $60 \mathrm{~min}$ proved to provide the optimal $\mathrm{PbI}_{2}$ layer for improving device photovoltaic performance. This resulting $\mathrm{PbI}_{2}$ wrapping layer, with a band gap $0.8 \mathrm{eV}$ greater than the $1.5 \mathrm{eV}$ bandgap of $\mathrm{MAPbI}_{3}$, results in a type-I alignment heterojunction to suppress trap induced recombination at the surface and grain boundaries (Fig. 6a-c). This ultimately increases the carrier lifetime, and enhances the device performance. Annealing at $150{ }^{\circ} \mathrm{C}$ for too long introduces excessive $\mathrm{PbI}_{2}$ and degrades the perovskite film and is detrimental to the device performance.

3.3.2 Conversion of extended imperfection to layered perovskite. The formation of layered perovskite between the grains of 3D perovskite is another effective approach for creating type-I band alignment at grain boundaries. ${ }^{116-119,137}$ The larger bandgap of layered perovskite is able to energetically wrap grains 
a

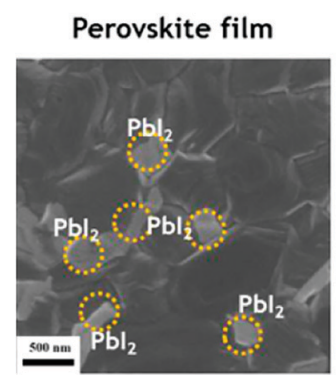

b

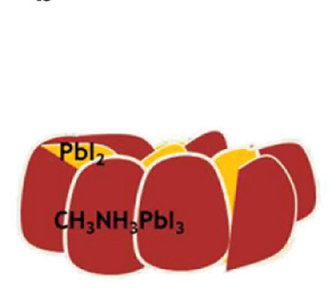

C

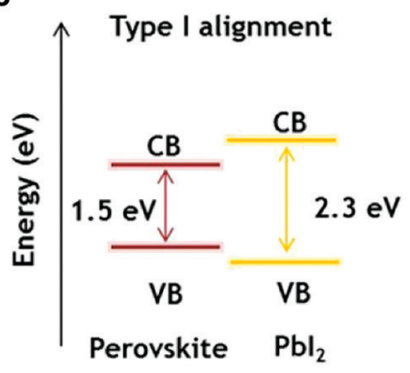

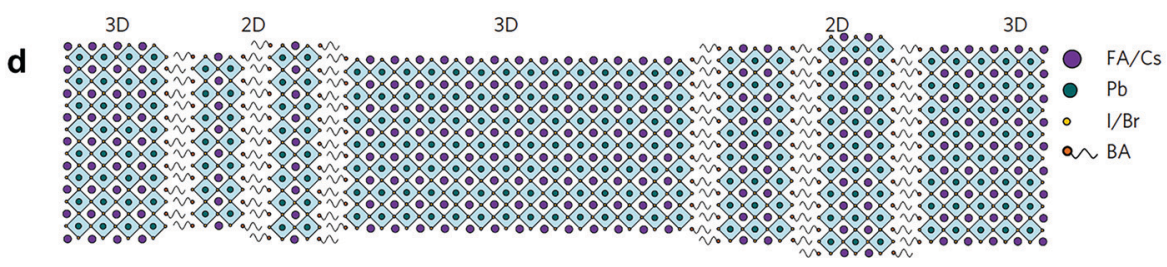
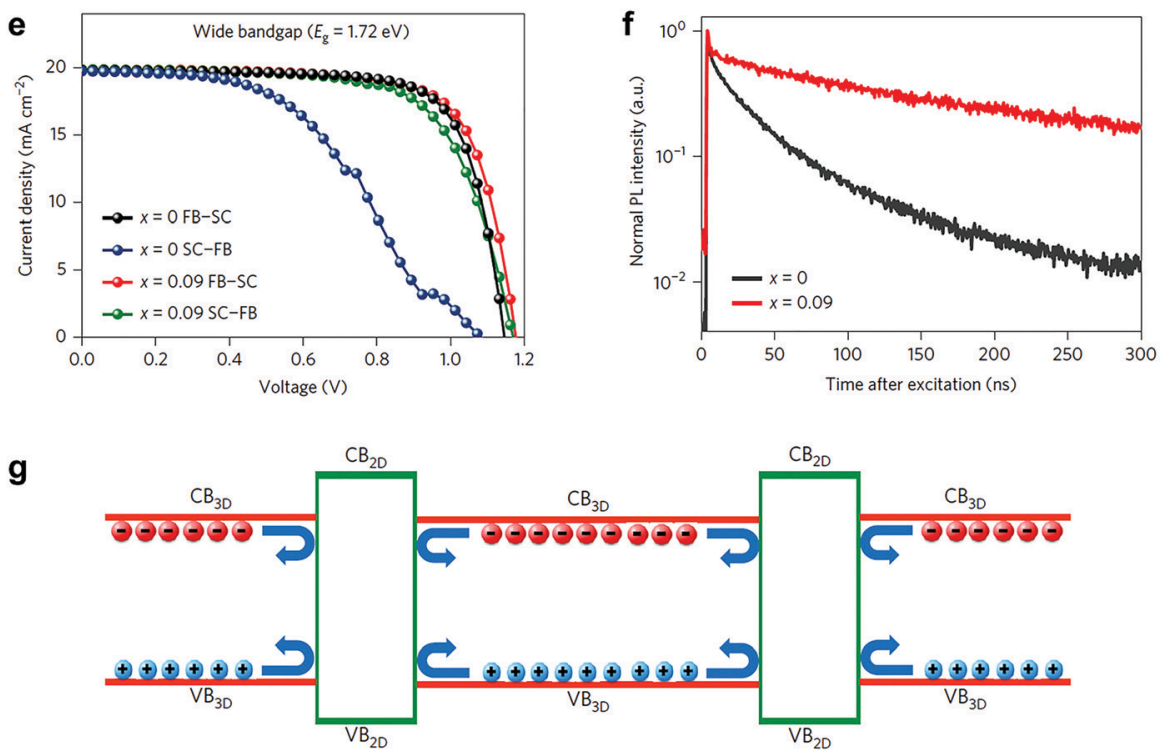

Fig. 6 Reduction of carrier recombination by type-I alignment. (a-c) $\mathrm{Pbl}_{2}$ passivation: (a) SEM, (b) schematic illustration, and (c) band alignment of $\mathrm{MAPb}_{3}$ film with $\mathrm{Pbl}_{2}$ wrapping the perovskite grain. Reprinted with permission from ref. 68. Copyright 2014 American Chemical Society. (d-g) Layered/ 3D perovskite heterostructures: (d) schematic illustration of the layered/3D perovskite heterostructure. (e) $J-V$ curves for perovskite solar cells using $1.72 \mathrm{eV}$ perovskite $\mathrm{BA}_{x}\left(\mathrm{FA}_{0.83} \mathrm{Cs}_{0.17}\right)_{1-x} \mathrm{~Pb}\left(\mathrm{I}_{0.6} \mathrm{Br}_{0.4}\right)_{3}$ with different amount of $\mathrm{BA}$ ratio, measured from forward bias (FB) to short-circuit (SC) and back again. (f) Time-resolved PL spectra of an $x=0$ film and an $x=0.09$ film. (g) Proposed electronic band offsets of the layered/3D heterojunction. Reproduced from ref. 117 with permission from Springer Nature, copyright 2017.

of the narrower bandgap 3D phase, establishing a type-I heterojunction at grain boundaries. Moreover, the conversion of defect rich grain boundaries into layered perovskite might also heal these defects to dramatically reduce defect density.

The layered/3D heterostructures with type-I band alignment have been investigated by incorporation of different layered perovskite precursors, such as $n$-butylammonium $\left(\mathrm{BA}^{+}\right),{ }^{116,117,137}$ phenethylammonium $\left(\mathrm{PEA}^{+}\right),{ }^{118,137}$ or aminovaleric acid iodide (AVAI). ${ }^{119}$ Wang et al. was the first to convert the grain boundary of $3 \mathrm{D}$ perovskite to layered perovskite for defect passivation. ${ }^{117}$ They mixed BA into $\mathrm{FA}_{0.83} \mathrm{Cs}_{0.17} \mathrm{~Pb}\left(\mathrm{I}_{0.6} \mathrm{Br}_{0.4}\right)_{3}$ perovskite precursor, ${ }^{117}$ and observed layered perovskite platelets standing upright between highly orientated 3D perovskite grains (Fig. 6d).
These layered/3D perovskite films had a significant increase in PL lifetime (Fig. 6f), revealing the formation of a layered phase can significantly reduce the non-radiative recombination at grain boundaries. ${ }^{117}$ Furthermore, Wang et al. proposed that lattice matching between the in-plane lead-halide sheets of layered perovskite and the lattice constant of the 3D phase allows for the self-assembly of the layered phase at the interface while avoiding the generation of defects responsible for deep level traps at grain boundaries, and the type I alignment further reduce the recombination at grain boundaries. ${ }^{117}$ Lee et al. reported the incorporation of large $\mathrm{PEA}^{+}$cations into $\left(\mathrm{FAPbI}_{3}\right)_{0.85}\left(\mathrm{MAPbBr}_{3}\right)_{0.15}$ was able to passivate the grain boundaries due to the formation of type-I band alignment, ${ }^{118}$ which was found to increase the PL 
intensity and improve the radiative recombination lifetime. ${ }^{118}$ Kelvin Probe Force Microscopy (KPFM) measurement shows that contact potential difference (CPD) between grain boundaries and the grain interior has been changed by the incorporation PEA. ${ }^{118}$ The conversion of extended surface to layered perovskite by incorporation of $\mathrm{BA}^{+}$and $\mathrm{PEA}^{+}$has also been reported capable of stabilizing the favorable black phase of $\mathrm{FASnI}_{3}$ perovskite and $\mathrm{FAPbI}_{3}$ perovskite, respectively. ${ }^{115,144}$ Besides modification of the perovskite precursor to attain layered perovskite for passivation, Lin et al. found spin coating the $n$-butylammonium iodide and $n$-Butylamine solution on 3D perovskite film could generate layered/3D stacking structures with layered perovskite still wrapping the perovskite grain boundaries and surface. ${ }^{116}$ Due to the poor conductivity of layered perovskite, it is important to tune the thickness of the layered phase to allow for tunneling at the perovskite/CTL interface to ensure good charge extraction. When the ratio of layered structure is too high, due to long reaction time $^{116}$ or high concentration of ammonium cations, ${ }^{118}$ the layered/3D heterostructures will become detrimental to device performance. $^{116,118}$

As mentioned previously, the use of ammonium cations for the passivation of $3 \mathrm{D}$ perovskite materials has been reported to result in two possible scenarios: (1) the conversion of extended imperfections to layered perovskite and (2) passivation of the defects by electrostatic interactions without forming a layered perovskite phase. Through comparing the effect of $\mathrm{NH}_{3} \mathrm{I}\left(\mathrm{CH}_{2}\right)_{4} \mathrm{NH}_{3} \mathrm{I}$ (C4), $\mathrm{NH}_{3} \mathrm{I}\left(\mathrm{CH}_{2}\right)_{8} \mathrm{NH}_{3} \mathrm{I}(\mathrm{C} 8)$, and $\mathrm{NH}_{3} \mathrm{I}\left(\mathrm{CH}_{2}\right)_{2} \mathrm{O}\left(\mathrm{CH}_{2}\right)_{2} \mathrm{NH}_{3} \mathrm{I}\left(\mathrm{EDBA}^{2+}\right)$, Zhao et al. found that the properties of the organic chain in ammonium cations play an important role to determine whether conversion could occur. ${ }^{114}$ Zhao et al. proposed that the unique anti-gauche isomerization of $\mathrm{C} 8$ raises the activation energy of the $3 \mathrm{D}$ to layered conversion, resulting in solely surface passivation without conversion to layered perovskite (Fig. 7a and Table 2). ${ }^{114}$
Even if a molecule has the same or shorter chain length compared to $\mathrm{C} 8$, like $\mathrm{EDBA}^{2+}$ or $\mathrm{C} 4$, Zhao et al. proposed that if it is locked in an anti-conformation without any possible gauche conformation, it lowers the activation energy for $3 \mathrm{D}$ to layered conversion resulting in an observed layered phase (Fig. 7b). This highlights the importance of molecular design beyond considering just the chain size to achieve passivation, either through solely surface passivation or conversion to a layered phase. These molecular design rules agree well with Jung et al.'s observation that $\mathrm{BA}^{+}$and $\mathrm{PEA}^{+}$inducing a layered/3D phase mixture but $\mathrm{OA}^{+}$, which is capable of anti-gauche isomerization, producing a pure 3D phase wrapped with $\mathrm{OA}^{+}{ }^{58}$ Similar to impact of conversion activation energy of $\mathrm{C} 4$ or $\mathrm{EDBA}^{2+}$, we suspect that the 3D-to-layered conversion by $\mathrm{PEA}^{+}$likely still has some energetic barrier that cannot be overcome at room temperature but can be overcome under thermal annealing. This might explain why surface treatment of PEAI without post annealing could obtain solely surface passivation effect without the formation of layered perovskite, and the 3Dto-layered perovskite conversion occurs after thermal annealing the PEAI coated perovskite films. ${ }^{122}$

\section{Suppression of ion migration via defect passivation}

It has been reported that the dominant point defects in perovskite materials create only shallow level traps as point defects resulting in deep level traps are difficult and unlikely to form. ${ }^{17-21}$ There are at least twelve point defects in the methylammonium lead triiodide $\left(\mathrm{MAPbI}_{3}\right)$ : three types of vacancies $\left(\mathrm{V}_{\mathrm{MA}}, \mathrm{V}_{\mathrm{Pb}}, \mathrm{V}_{\mathrm{I}}\right)$, three types of interstitial sites $\left(\mathrm{MA}_{\mathrm{i}}, \mathrm{Pb}_{\mathrm{i}}, \mathrm{I}_{\mathrm{i}}\right)$, two types of cation substitutions $\left(\mathrm{MA}_{\mathrm{Pb}}, \mathrm{Pb}_{\mathrm{MA}}\right)$, and four types of antisite substitutions $\left(\mathrm{MA}_{\mathrm{I}}, \mathrm{Pb}_{\mathrm{I}}, \mathrm{I}_{\mathrm{MA}}, \mathrm{I}_{\mathrm{Pb}}\right)$ where in the latter

\section{a}

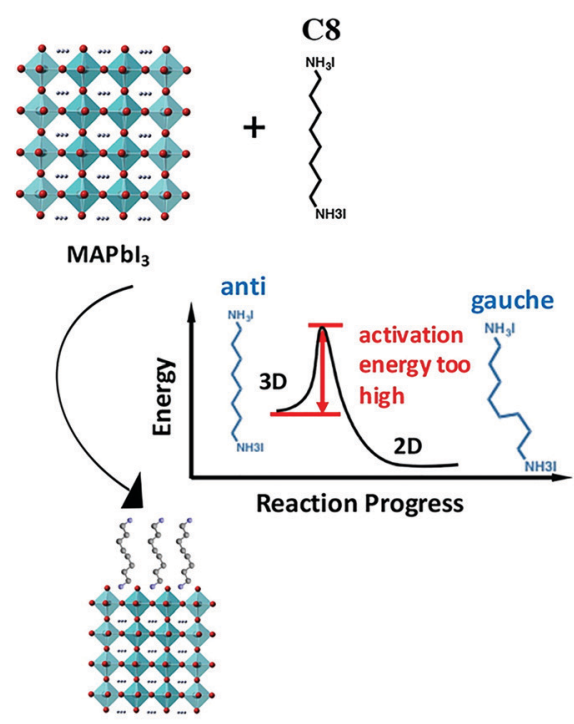

b

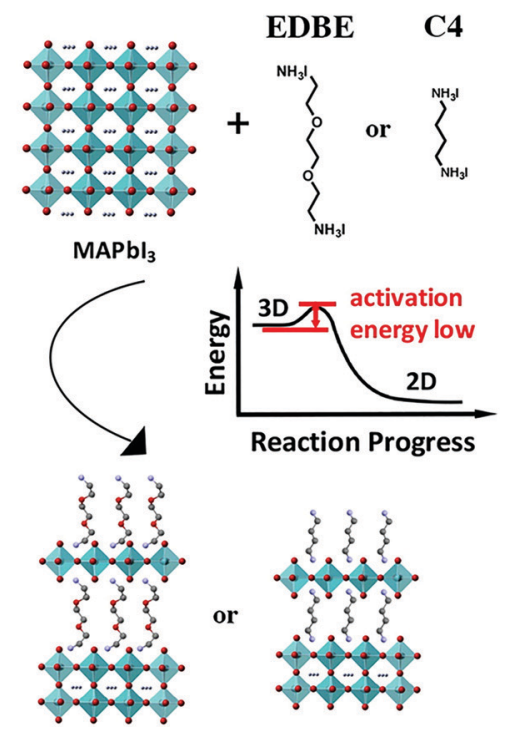

Fig. 7 Extended defect passivation versus 3D-to-layered perovskite conversion. (a) Schematic demonstration of why C8 stays only passivate the surface or grain boundaries without conversion to layered perovskite, while (b) EDBE and C4 treatment can induce respective 2D hybrid perovskite formation. Adapted with permission from ref. 114. Copyright 2016 American Chemical Society. 

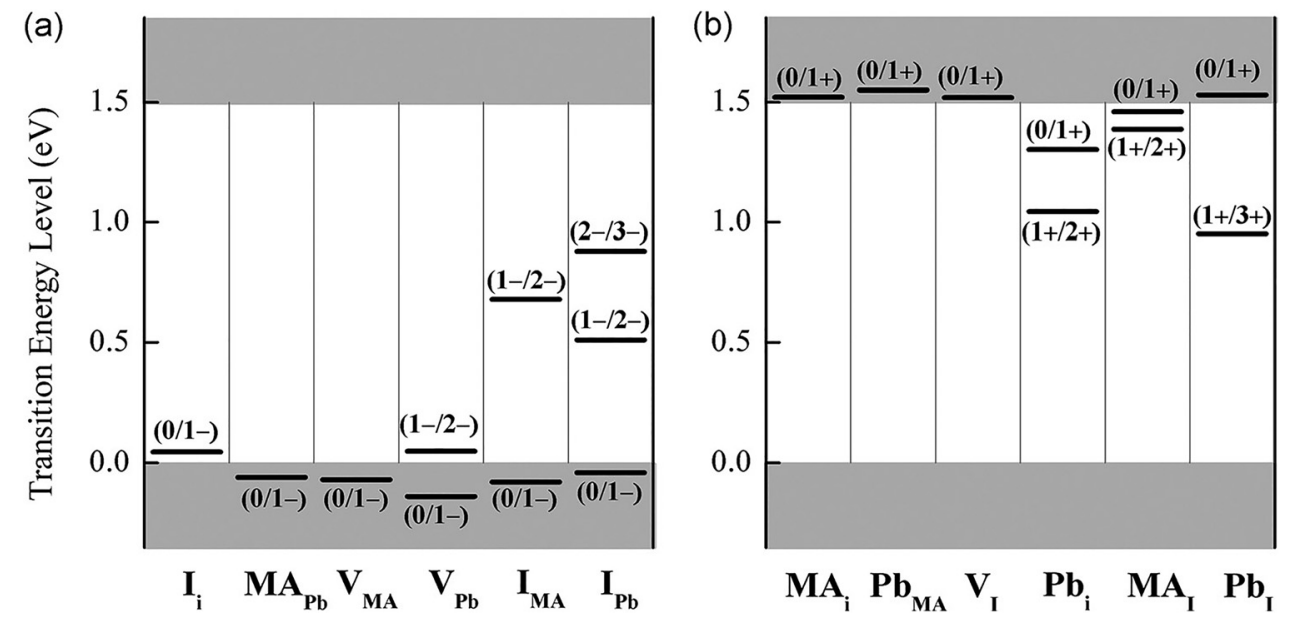

Fig. 8 The transition energy levels of (a) intrinsic acceptors and (b) intrinsic donors in MAPbl 3 . Reproduced from ref. 18 with permission from AlP Publishing, copyright 2014.

cases $A_{B}$ indicates that $A$ is substituted by B. ${ }^{17-19}$ As shown in Fig. 8, Yin et al. found that the point defects with low formation energy create shallow level traps, such as $\mathrm{MA}_{\mathrm{i}}, \mathrm{V}_{\mathrm{Pb}}, \mathrm{MA}_{\mathrm{Pb}}, \mathrm{I}_{\mathrm{i}}, \mathrm{V}_{\mathrm{I}}$, and $\mathrm{V}_{\mathrm{MA}}$, while all defects that induce deep level traps have high formation energy, such as $\mathrm{I}_{\mathrm{Pb}}, \mathrm{I}_{\mathrm{MA}}, \mathrm{Pb}_{\mathrm{i}}$, and $\mathrm{Pb}_{\mathrm{I}}{ }^{18}$ The shallow trap states close to the conduction or valence band edges can induce either n-type or p-type self-doping of perovskites. ${ }^{18}$ Even though the shallow level point defects do not contribute to non-radiative recombination, their ionic nature enables them to migrate within an electric field. The accumulation of those charged defects at each side of OIHP layers results in a high charge density capable of inducing PL quenching effects. ${ }^{128}$ The redistribution of mobile defects could cause band bending, ${ }^{63}$ current density-voltage $(J-V)$ hysteresis, ${ }^{37,39-43}$ reaction with function layers beyond OIHP, ${ }^{48-52}$ or phase segregation. ${ }^{44-47}$ In addition to Yuan et al. reported that with an applied electric field $\mathrm{MA}^{+}$and $\mathrm{I}^{-}$were capable of migration within OIHP films. ${ }^{145,146}$ Shao et al. also proved that grain boundaries are the primary migration channel for mobile ions, ${ }^{64}$ highlighting the importance of future investigations on the suppression of ion migration via passivation of extended defects. Moreover, light induced phase segregation of mixed halide OIHP film is also reported to be connected to ion migration under illumination. ${ }^{44-46}$ deQuilettes et al. observed the migration of $\mathrm{I}^{-}$in $\mathrm{MAPbI}_{3}$ film under illumination. ${ }^{147}$ They proposed that the trapped photo-excited electrons in vacancies, particular near the surface, produced an electric field capable of driving the migration of halides. ${ }^{147}$ The migration of ions is reported to increase significantly under illumination due to a lower activation energy for ion migration. ${ }^{42,148}$ Therefore, it is important to suppress the migration of point defects for efficient and stable perovskite solar cells.

\subsection{Band bending due to migration of point defects}

The migration of mobile ions could cause band bending due to electric field screening effect or local doping/de-doping effect, and thus impact the charge extraction efficiency. Under the electric field, the positively charged point defects, such as $V_{I}$ and $\mathrm{MA}_{\mathrm{i}}$, migrate along the electric field direction, while negatively charged point defects, such as $V_{M A}$ and $I_{i}$, migrate in the opposite direction. ${ }^{39,40,63,149}$ This creates accumulation of charged defects at perovskite/CTL interface. It has been proposed that this ion migration could create an additional electric field across the perovskite film and cause electric field screening effect. ${ }^{39,40,63,149}$ This leads to change of net built-in electric field and the carrier transport and extraction efficiency. Due to the ionic nature of perovskite materials, the accumulation of charged point defects at interfaces may also result in an unintentional doping effect, ${ }^{18,149-151}$ which could alter the band bending of interfaces. In order to keep the material electrically neutral, the positively charged mobile defects accumulated at interface will attract equivalent electrons. Thus local excessive ions serve as a donor to chemically dope the perovskite surface to n-type. Similarly, the accumulation of negative charged mobile ions near interface causes p-type doping. Yin et al. found that the dominant defects in $\mathrm{MAPbI}_{3}$ are p-type $\mathrm{V}_{\mathrm{Pb}}$ and n-type $\mathrm{MA}_{\mathrm{i}}$ with low defect formation energy, and the $\mathrm{MAPbI}_{3}$ could change from good p-type, intrinsic to good n-type by controlling the growth condition to tune the amounts of dominant defects. ${ }^{18}$ After device fabrication in dark condition, the ions migration could already occur under built-in electric field to partially compensate the built-in electric field.

During formation of perovskite solar cells, a build-in electric field is already generated even in dark condition by the work function difference between anode and cathode pointing from ETL to HTL. This field drives the migration of positively charged point defects, such as $\mathrm{V}_{\mathrm{I}}$ and $\mathrm{MA}_{\mathrm{i}}$ toward the HTL, while negatively charged point defects, such as $\mathrm{V}_{\mathrm{MA}}$ and $\mathrm{I}_{\mathrm{i}}$, migrate in the opposite direction in dark condition (Fig. 9a). ${ }^{39,40,63,149}$ The accumulation of these charged ions at perovskite/CTL interfaces has been proposed to create an additional electric field across the perovskite film that is opposite to the built-in electric field, which hinders the collection of photogenerated charges. ${ }^{39,40,63,149}$ Deng et al. reported the light soaking could induce a more favorable 


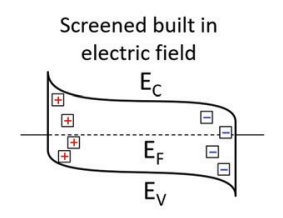

b
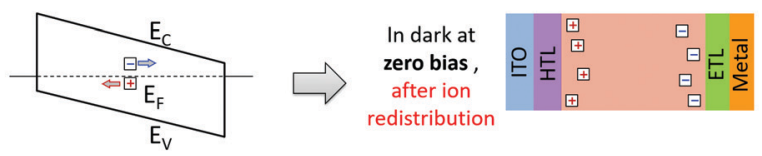

Light induced self-poling
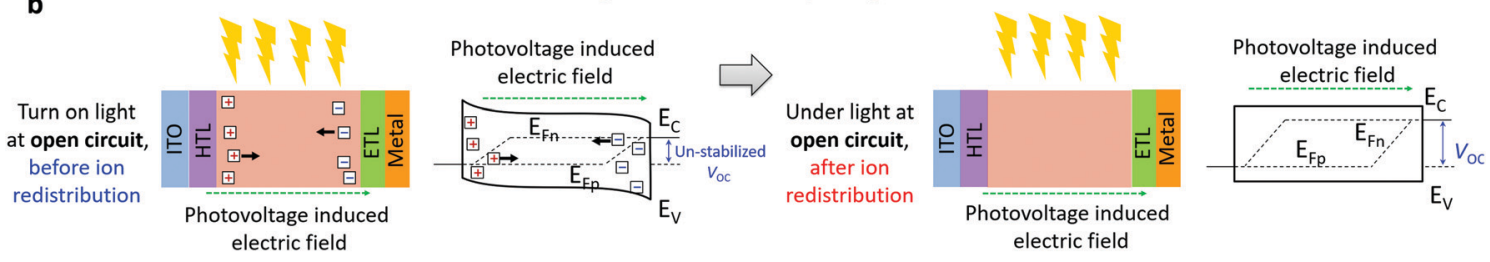

c

Hysteresis due to ion migration
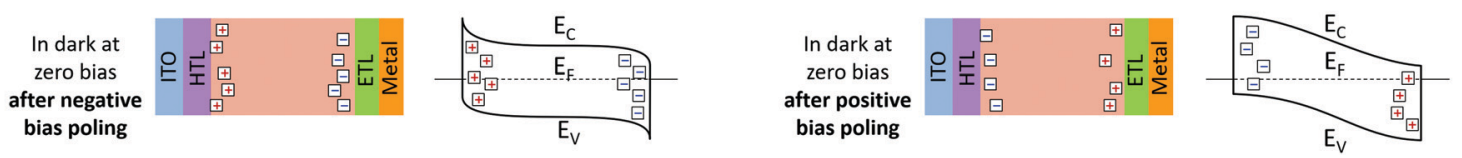

Fig. 9 Sketch of band bending due to ion migration in PSCs. (a) Band configuration of PSCs in dark at open circuit condition before and after ion redistribution under build-in electrical field. (b) Band configuration of PSCs when turn on the light at open circuit condition before and after ion redistribution under photovoltage. (c) Band configuration of PSCs in dark at zero bias after negative bias poling or after positive bias poling.

$\mathrm{p}-\mathrm{i}-\mathrm{n}$ structure due to ion redistribution under photovoltage induced electrical field, which enhances the charge collection efficiencies. ${ }^{150}$ At open circuit condition under illumination, the additional photovoltage induced field points from HTL to ETL, which drives the migration of positively charged point defects toward the ETL, while negatively charged point defects migrate into the opposite direction. After ion redistribution, in ideal case, there is no ion accumulation near interface at open circuit condition under illumination, because the net built-in electric field is zero (Fig. 9b). This ion redistribution under illumination eliminates the detrimental effect of ion accumulation on charge collection.

Furthermore, although not detailed in this review, ion migration has been broadly reported to induce $J-V$ hysteresis in PSCs. ${ }^{37-43}$ The $J-V$ hysteresis in PSCs typically presents itself as a difference in the shape of $J-V$ curves when the measurement is done scanning from reserve bias and from forward bias, typically the reverse scan from $V_{\mathrm{OC}}$ to $0 \mathrm{~V}$ shows better $\mathrm{FF}, V_{\mathrm{OC}}$, and PCE if there is $J-V$ hysteresis in PSCs. ${ }^{37-43}$ The $J-V$ hysteresis has been reported to be influenced by the direction/rate/ range of scanned voltage, device preconditions, device configuration, and charge extraction efficiency of CTL. ${ }^{37-43}$ Xing et al. measured the ion drift velocity in $\mathrm{MAPbI}_{3}$ polycrystalline films under illumination, reporting that the ion migration across a $\mathrm{MAPbI}_{3}$ thin film in PSCs is on the timescale of around $1 \mathrm{~s}^{42}$ Due to the slow response of ion migration under electric field, the amount of accumulated mobile defects is different under the same applied bias during the forward and reverse scan (Fig. 9c). More details about the change of band configuration due to ion migration under illumination or in dark can be found in ref. 40. Therefore, the device functions with different band bending conditions due to ion migration between forward and reverse scans, resulting in $J-V$ hysteresis. ${ }^{37-43}$ As reviewed by Kang et al., ${ }^{43} \mathrm{~J}-V$ hysteresis in PSCs is also related with capacitive current, which can be generated by both ion migration and nonradiative recombination near interfaces. Thus the accumulation of ionic charges at interfaces may interrupt the extraction of photo-generated carriers to build a large capacitance at the interface and induce hysteretic behavior.

\subsection{Reaction at interface due to ion migration}

Migration of mobile ions to either the perovskite/ETL or perovskite/HTL interfaces may result in an undesirable reaction between mobile ions and the CTLs or metal electrode. Carrillo et al. observed a chemical reaction between the oxidized spiro-OMeTAD ${ }^{+}$and $\mathrm{I}^{-}$ions resulting in the formation of a neutral spiroMeOTAD-iodide complex (Fig. 10a-c).$^{48}$ Absorption spectroscopy shows that the neutral spiro-OMeTAD film has a characteristic band between $300-420 \mathrm{~nm}$, and the oxidized spiro-OMeTAD ${ }^{+}$ has an additional polaronic band around $500 \mathrm{~nm}$ (Fig. 10d). When the oxidized spiro-OMeTAD ${ }^{+}$is treated with $\mathrm{I}^{-}$ions, such as from MAI, the absorption intensity of polaronic band reduces significantly. In the cycled $I-V$ measurement including positive poling for $5 \mathrm{~min}$, they found a redox peak in PSCs with a device structure of $\mathrm{FTO} / \mathrm{TiO}_{2} / \mathrm{MAPbI}_{3} /$ spiro-OMeTAD/Au. ${ }^{48}$ This redox peak is proposed to correlate with the chemical reaction between oxidized spiro-OMeTAD ${ }^{+}$and $\mathrm{I}^{-}$ions. $^{48}$ The irreversible chemical reaction at the perovskite/spiro-OMeTAD interface progressively reduces the HTL conductivity and deteriorates solar cell performance. Using kelvin probe force microscopy, this reaction was determined to also result in the change of local contact potential difference (LCPD) at the oxidized spiroMeOTAD layer 
a

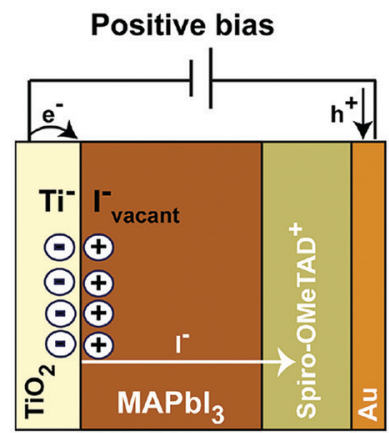

b

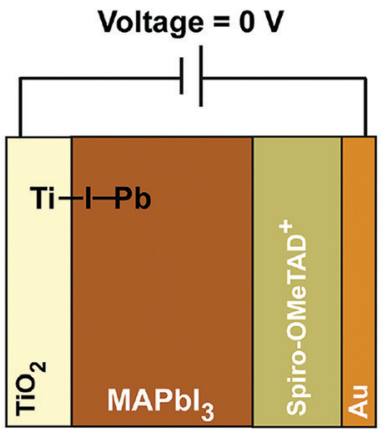

C

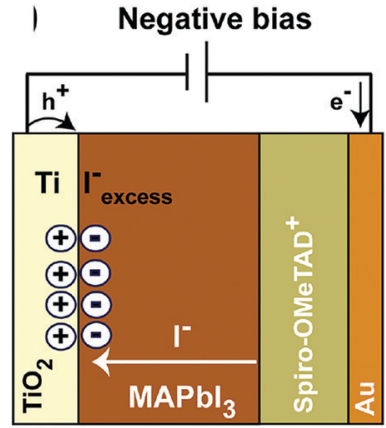

d

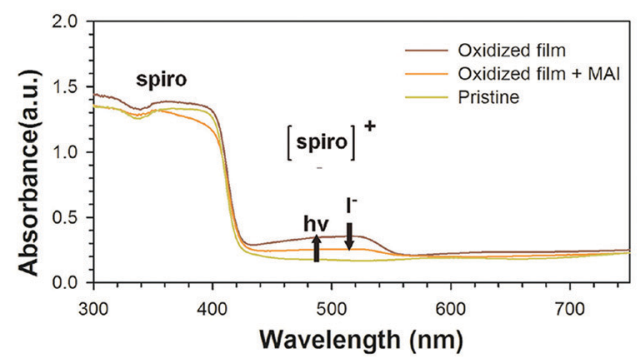

e

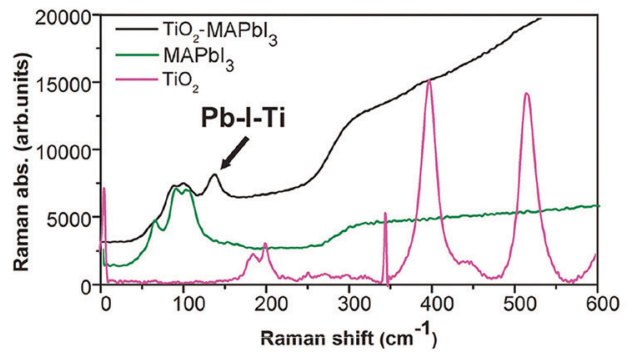

f Step 1

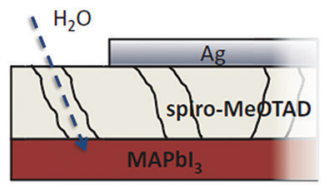

Step 4

Surface diffusion of iodine-containing compound

Step 2
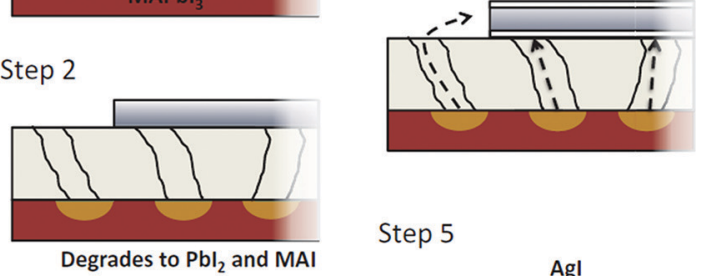

Step 5

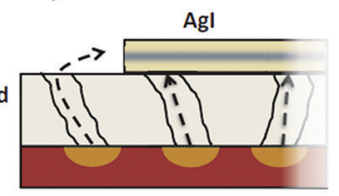

Fig. 10 Reaction at CTL interfaces due to ion migration. (a-c) Schematic diagram of iodide migration and chemical species present at the interfaces. (a) Migration of iodine ions to HTL under positive bias and reaction between oxidized spiro-OMeTAD ${ }^{+}$and I- ions to form neutral spiroMeOTAD-iodide complex. (b) Under zero-bias the neutral case appears. (c) Under negative-bias spiro-OMeTAD only partially recovers to its oxidized state, and iodide ions accumulate at the $\mathrm{TiO}_{2}$ /perovskite interface. (d) Absorption spectra of films of pristine spiro-OMeTAD, oxidized spiro-OMeTAD layers, and oxidized spiro-OMeTAD layers with MAI. (e) Raman spectra illustrating the formation of Ti-I-Pb bonds upon reaction between $\mathrm{MAI}^{2}$ and Pbl 2 . Reproduced from ref. 48 with permission from John Wiley and Sons, copyright 2016. (f) Schematic illustration of a proposed mechanism of Agl formation by the migration of MAI through the spiro-MeOTAD layer, aided by pinholes. Reproduced from ref. 52 with permission from John Wiley and Sons, copyright 2015.

after light illumination. ${ }^{49}$ Kim et al. found this reaction between oxidized spiro-OMeTAD ${ }^{+}$and mobile $\mathrm{I}^{-}$ions could also be activated by thermal stress when the device temperature is elevated to $85{ }^{\circ} \mathrm{C}^{50}$ The mobile $\mathrm{I}^{-}$ions not only can react with the oxidized spiro-OMeTAD $^{+}$but also some metal electrodes, such as silver. ${ }^{51,52}$ Kato et al. observed that silver electrodes for $\mathrm{MAPbI}_{3}$ cells will turn yellow within days after device fabrication due to the formation of AgI, and proposed that it was a product of the electrode reacting with MAI that had migrated through the spiro-MeOTAD layer, aided by pinholes (Fig. 10f). ${ }^{52}$ At the perovskite/ $/ \mathrm{TiO}_{2}$ interface, Carrillo et al. proposed an ionic reaction between the $\mathrm{MAPbI}_{3}$ and $\mathrm{TiO}_{2}$ to form weak Ti-I-Pb bonds (Fig. 10e), which facilitates interfacial movement of iodine ions and produces a reversible capacitive current at $\mathrm{MAPbI}_{3} / \mathrm{TiO}_{2}$ interface. ${ }^{48}$

\subsection{Photoinduced phase segregation due to ion migration}

Besides the impact on photovoltaic performance through band bending, ion migration has also been reported to be involved in photoinduced phase segregation in mixed halide OIHP films. ${ }^{44-46}$ Hoke et al. found that the initial photoluminescence (PL) of $\operatorname{MAPb}\left(\mathrm{Br}_{x} \mathrm{I}_{1-x}\right)_{3}$ thin films show a monotonic increase in the band gap with increasing Br content (Fig. 11a); however, an additional PL peak at $1.68 \mathrm{eV}$ appears and grows in intensity under illumination for compositions of $\mathrm{MAPb}\left(\mathrm{I}_{1-x} \mathrm{Br}_{x}\right)_{3}$ thin films with $0.2<x<1$ (Fig. 11b). ${ }^{44}$ X-ray diffraction (XRD) shows peak splitting for $\operatorname{MAPb}\left(\mathrm{Br}_{0.6} \mathrm{I}_{0.4}\right)_{3}$ films with light soaking (Fig. 11c), as well as the recovery of the original diffraction pattern after the films are left in the dark. ${ }^{44}$ This indicates the illumination of $\operatorname{MAPb}\left(\mathrm{Br}_{0.6} \mathrm{I}_{0.4}\right)_{3}$ films results in the formation of separate I-rich and Br-rich domains. As shown in Fig. 11d, Bischak et al. proposed that under illumination the segregation into a Br-rich and I-rich phase provides the lowest free energy for the $\operatorname{MAPb}\left(\mathrm{Br}_{x} \mathrm{I}_{1-x}\right)_{3}$ film, while the single phase $\operatorname{MAPb}\left(\mathrm{Br}_{x} \mathrm{I}_{1-x}\right)_{3}$ is the lowest free energy state in dark conditions. ${ }^{45}$ They observed phase segregation of mixed halide OIHP film under illumination by cathodoluminescence (CL) imaging (Fig. 11e and f), and reported the formation of I-rich 

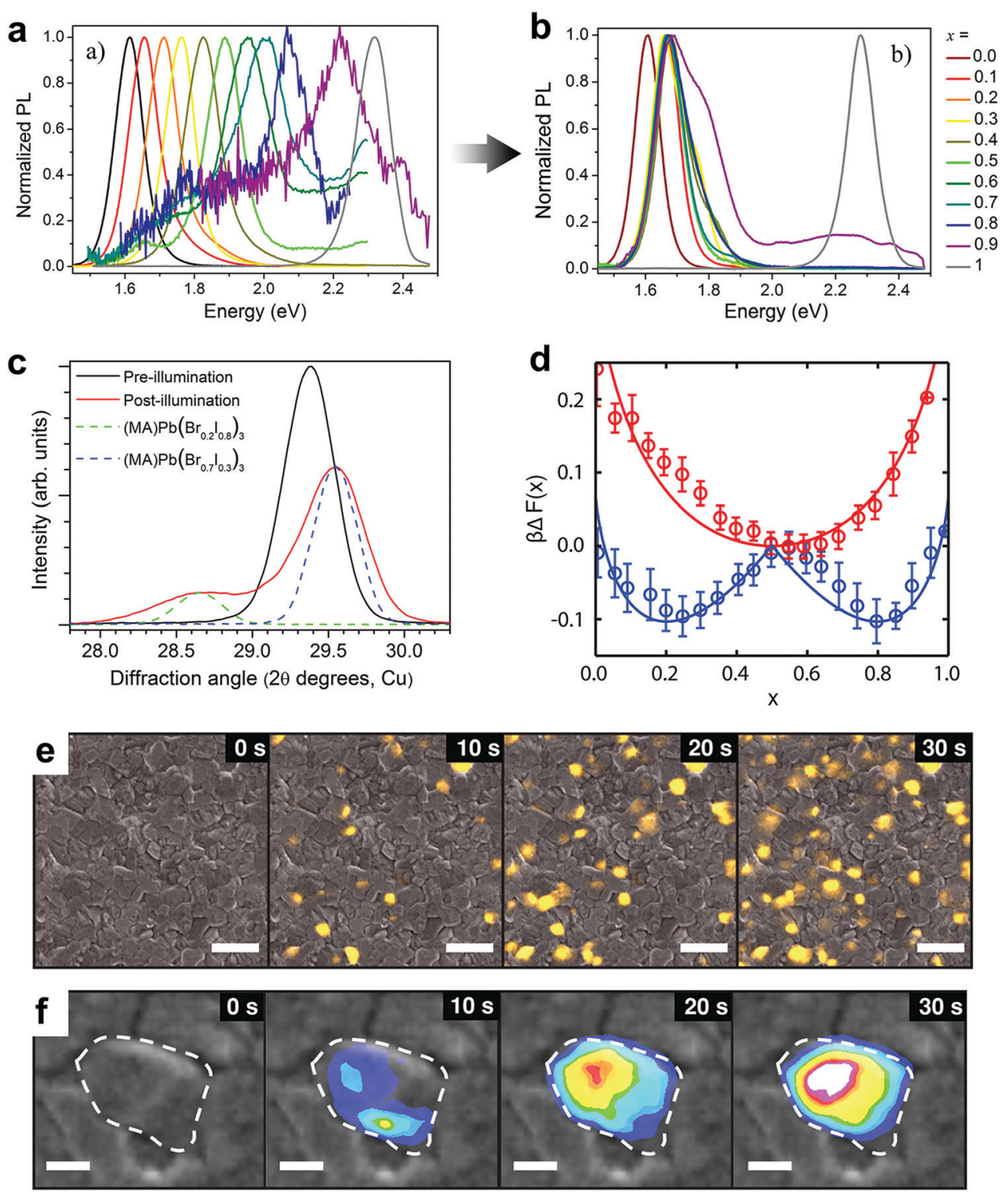

Fig. 11 Photoinduced phase segregation. Normalized $\mathrm{PL}$ spectra of $\mathrm{MAPb}\left(\left.\mathrm{Br}_{x}\right|_{1-x}\right)_{3}$ films before (a) and after (b) illuminating for $5-10$ min under $457 \mathrm{~nm}$

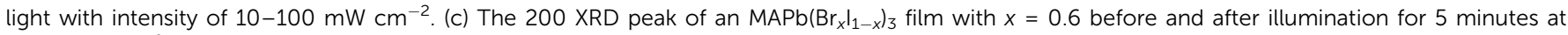
$\sim 50 \mathrm{~mW} \mathrm{~cm}^{-2}$ white light. XRD patterns of an $x=0.2$ film and an $x=0.7$ film are included for comparison. Reproduced from ref. 44 with permission from The Royal Society of Chemistry. (d) Free energies per unit cell for $\mathrm{MAPb}\left(\mathrm{Br}_{x} l_{1-x}\right)_{3}$ with varying composition in the ground (red) and photoexcited (blue) states, computed from MD simulations (circles) and mean field theory (solid lines). (e) CL image series of $M A P b\left(B r_{x} l_{1-x}\right)_{3}$ film with $10 \mathrm{~s}$ of light soaking between each image. The scale bars are $2 \mu \mathrm{m}$. (F) Enlarged $\mathrm{CL}$ image series of $\mathrm{MAPb}\left(\left.\mathrm{Br}_{x}\right|_{1-x}\right)_{3}$ film and the color scale indicates iodide-rich $\mathrm{CL}$ intensity. The scale bar is $200 \mathrm{~nm}$. Reprinted with permission from ref. 45. Copyright 2017 American Chemical Society.

clusters at grain boundaries. ${ }^{45}$ Bischak et al. also suggested that the localized strain due to the interaction of photoexcited carriers with the ionic OIHP lattice is able to facilitate the halide phase separation being observed. ${ }^{45}$ Wide bandgap PSCs with mixed halide OIHP materials are integral for application in tandem solar cells, and the ion migration induced phase segregation hinder their performance in tandem devices.

\subsection{Strategies to suppress ion migration}

Passivation of grain boundaries has not only been critical in improving device performance, but is also an effective method to suppress ion migration. Shao et al. was the first to elucidate that grain boundaries provide the dominant pathway for ion migration in polycrystalline $\mathrm{MAPbI}_{3}$ films. ${ }^{64}$ Conductive atomic force microscopy measurements demonstrated much stronger $J-V$ hysteresis at the boundaries than at the grain interiors, while energy-dispersive X-ray spectroscopy also revealed a gradient in iodine concentration along grain boundaries after electric poling that is not present at grain interiors. ${ }^{64}$ Shao et al. also reported that by passivating grain boundaries with PCBM it was possible to deter ion migration and eliminate the corresponding $J-V$ hysteresis. ${ }^{64}$ Abdi-Jalebi et al. utilized KI to passivate the surfaces and grain boundaries and mitigate the photoinduced ion migration in perovskite. ${ }^{47}$ They found that 
the external photoluminescence quantum efficiency (PLQE) of $\left(\mathrm{Cs}_{0.06} \mathrm{FA}_{0.79} \mathrm{MA}_{0.15}\right) \mathrm{Pb}\left(\mathrm{I}_{0.85} \mathrm{Br}_{0.15}\right)_{3}$ thin films slowly increased under illumination, indicative of photoinduced halide migration, while the PLQE of KI passivated samples are stabilized due to suppressed halide migration at the grain boundaries. Passivation by $\mathrm{KI}$ of the extended imperfections in wide bandgap compositions $\left(\mathrm{Cs}_{0.06} \mathrm{FA}_{0.79} \mathrm{MA}_{0.15}\right) \mathrm{Pb}\left(\mathrm{I}_{1-y} \mathrm{Br}_{y}\right)_{3}$, with $y=0.4$ or even $y=0.6$, also proves successful in suppressing halide segregation with no shift in PL. ${ }^{47}$ As proposed by Abdi-Jalebi et al., the function of KI can be two-fold. ${ }^{47}$ The first is that excess $\mathrm{I}^{-}$introduced can help fill $\mathrm{I}^{-}$vacancies and thus eliminate pathways for ion migration. Additionally, the $\mathrm{K}^{+}$ cations can form an ionic bond with undercoordinated halides or adsorb at negatively charged defects of the surface and grain boundaries, again either eliminating pathways for ion migration or raising the activation energy for ion migration to occur. It has also recently been proposed by Son et al. that $\mathrm{K}^{+}$is calculated to be thermodynamically favored to reside within interstitial sites and mitigate the formation of ion migration and Frenkel defects; ${ }^{108}$ however, experimental evidence of $\mathrm{K}^{+}$ occupying interstitial sites within the bulk of grains has yet to definitively reported. ${ }^{107-111}$

In addition to fullerenes' efficient passivation discussed earlier, the coating of surfaces and filling of grain boundaries with these large and immobile fullerenes organic molecules could also sterically hinder the ion migration at grain boundaries by physically blocking mobile ions. ${ }^{64}$ In the case of fullerene and its derivatives (PCBM included), the molecules are far too large to be incorporated into the lattice. The surface treatment of PCBM and the following thermal annealing induces the diffusion of PCBM into grain boundaries to block ion migration. Formation of layered perovskite also has been reported to significantly reduce the hysteresis by providing an effective physical barrier to suppress the ion migration at grain boundaries. ${ }^{117}$

The composition engineering of OIHP materials has also been reported as a successful approach to increase the activation energy of point defect formation and reduce the possible ion migration. Sargent et al. proposed that the local lattice strain in $\mathrm{MAPbI}_{3}$ and $\mathrm{FAPbI}_{3}$ induce the formation of point defects. ${ }^{71}$ When FA and I are partially replaced by Cs/MA and $\mathrm{Br}$, respectively, simulations based on density functional theory showed that the $\mathrm{PbI}_{2}$ vacancy formation energy is increased more than threefold, resulting in reducing the vacancy concentration by a factor of $\sim 10^{9} .{ }^{71}$ Negligible hysteresis in PSCs with mixed cation/halide perovskites, compared to either $\mathrm{MAPbI}_{3}$ and $\mathrm{FAPbI}_{3}$, further demonstrates the impact of increased defect formation energy on ion migration. ${ }^{71}$

Ion migration could also be accelerated by the presence of trapped charges due to the reduced activation energy for ion migration. ${ }^{108,152,153}$ Birkhold et al. found that the migration rate is increased after injection of carriers under electric field. ${ }^{153}$ Lin et al. discovered that excess holes and excess electrons accelerate the migration of cations and anions, respectively, by lowering the activation energy for ion migration within organic-inorganic halide perovskite films. ${ }^{152}$ The trapping of photo-excited charges by electronic traps due to the surface defect of perovskite materials can then facilitate ion migration and the generation of bulk defects. Moreover, the point defects inside the bulk crystal should be able to reach the crystal surface under an electrical field. Therefore, passivation of the extended defects could avoid trapped charges and suppress ion migration, ultimately acting to passivate the bulk of OIHP materials as well. Efficient charge extraction by selection of CTL could also reduce the amount of trapped charges to suppress ion migration. For example, doping the $\mathrm{TiO}_{2}$ by lithium salts, ${ }^{154}$ chlorine ions, ${ }^{134}$ or replacing the $\mathrm{TiO}_{2}$ by $\mathrm{SnO}_{2}$ as the $\mathrm{ETL}^{155}$ have been reported to effectively suppress ion migration and eliminate the $J-V$ hysteresis in $\mathrm{n}-\mathrm{i}-\mathrm{p}$ perovskite solar cells.

\section{Impact of imperfection passivation on material and device stability}

Commercial silicon photovoltaic modules have proven to be stable for more than ten years, while most encapsulated PSCs barely last over six months under realistic operating conditions. ${ }^{156}$ It has been generally observed that moisture, oxygen, light, and elevated temperature can induce the accelerated degradation of perovskite solar cells. ${ }^{85,157-161}$ Moisture-, oxygen-, and lightinduced degradation of PSCs under illumination have all been reported to be accelerated by the presence of trapped charges. ${ }^{85,157-161}$ Therefore, passivation of extended and point imperfections has been demonstrated to significantly improve the stability of OIHP materials and devices.

Organic-inorganic halide perovskite films have been well documented to be sensitive to moisture. ${ }^{157,158}$ The reaction of $\mathrm{CH}_{3} \mathrm{NH}_{3} \mathrm{PbI}_{3}$ with moisture in dark has been shown to form a reversibly hydrated product. ${ }^{157}$ In the event that the entire film is converted to the hydrated product, any excess water could result in irreversible degradation. ${ }^{157}$ Under illumination, Ahn et al. found that trapped charges at the surface and grain boundaries facilitate the formation of highly volatile $\mathrm{CH}_{3} \mathrm{NH}_{2}$, which once evaporated can trigger an irreversible degradation of perovskite materials under moisture. ${ }^{158}$ Under dark condition, Ahn et al. found that the trapped charges from corona discharge or electrical injection can also accelerate the irreversible moistureinduced degradation of perovskites. ${ }^{158}$ Wang et al. found that the defective grain boundaries with a high density of trapped charges are the most vulnerable sites for moisture-induced degradation. ${ }^{162}$

Illumination of PSCs in the presence of oxygen is also capable of inducing rapid degradation. ${ }^{85,159-161}$ It is noted that the degradation rate of PSCs is greatly reduced when exposed only to oxygen without illumination. ${ }^{161}$ However, accelerated degradation of PSCs in dark conditions with oxygen present is still reported under applied current and electrical bias. ${ }^{161}$ This accelerated oxygen-induced degradation of PSCs under illumination is initiated by the reaction between molecular oxygen, $\mathrm{O}_{2}$, and photo-excited electrons to generate superoxide, $\mathrm{O}_{2}{ }^{-}$. The reaction between $\mathrm{O}_{2}{ }^{-}$and OIHP materials has been well reported to induce degradation by forming $\mathrm{PbI}_{2}, \mathrm{I}_{2}, \mathrm{H}_{2} \mathrm{O}$, and $\mathrm{CH}_{3} \mathrm{NH}_{2}{ }^{85,159,160}$ Therefore, the trapped charges can accelerate 
the oxygen-induced degradation of PSCs through the formation of $\mathrm{O}_{2}{ }^{-}$. Furthermore, it has been reported that PSCs operated under short-circuit conditions, rather than open circuit, show improved stability when exposed to light and oxygen. ${ }^{160}$ This is because the oxygen-induced photodegradation is retarded by extracting the photogenerated electrons before they can react with oxygen to form $\mathrm{O}_{2}{ }^{-159,160}$

The light induced degradation of perovskite solar cells is related with excess charge carrier. Lin et al. found that HTLcovered and ETL-covered $\mathrm{MAPbI}_{3}$ films degrade faster than the $\mathrm{MAPbI}_{3}$ films covered by an insulating layer. ${ }^{152}$ Temperaturedependent conductivity measurements determined when covered by a CTL, $\mathrm{MAPbI}_{3}$ films have a smaller activation energy $\left(E_{\mathrm{a}}\right)$ for ion migration than the $\mathrm{MAPbI}_{3}$ films covered by an insulating layer. ${ }^{152}$ Therefore, both excess electrons and excess holes under illumination could reduce the $E_{\mathrm{a}}$ for ion migration within OIHP materials, accelerating the degradation of PSCs. ${ }^{152}$ This also explains why the PSCs demonstrate faster degradation rate when operated at open circuit conditions with more excess charge carriers, compared to maximum power point conditions.

Due to trapped charges demonstrating the ability to accelerate moisture-, oxygen-, and light-induced degradation in PSCs under operating conditions, reducing the density of trapped charges by passivation of imperfections enhances the stability perovskite devices. The density of trapped charges can be reduced by two approaches: (a) reduce the trap density by passivating deep level traps and (b) reduce the density of free photo-excited charges by efficient charge extraction. Passivation of deep level traps by coordinate bonding, ionic bonding, or the conversion of extended imperfections to wide bandgap materials have all been deemed capable of effectively reducing the trap density in PSCs, as discussed in Section 3. The suppression of point defect migration can avoid adverse band bending to maintain the efficient extraction of charges and prevent excess carriers in OIHP films, as discussed in Section 4. Therefore, imperfection passivation and suppression of ion migration can significantly improve the stability of PSCs by reducing the density of trapped charges.

Conversion of extended imperfections to layered perovskite has been reported to improve the stability of perovskite solar cells. The layered perovskites demonstrate better moisture stability than their 3D counterparts, ${ }^{163-165}$ which has been attributed to the improved hydrophobicity due to the longer alkyl chains of $\mathrm{BA}^{+}$and $\mathrm{PEA}^{+}$and the more energy necessary for them to leave the structure, compared to $\mathrm{MA}^{+} .^{163-165}$ Therefore the conversion of defective surfaces and grain boundaries to layered perovskite is suspected to mitigate the loss of volatile MA and $\mathrm{I}_{2}$ species and improve the device stability. ${ }^{163-165}$ Coupling the elimination of defects via conversion discussed in Sections 3.2.1 and 3.3.2 with the suppressed ion migration reported for layered perovskites, the conversion of the surface and grain boundaries of OIHP thin films to layered perovskite ofproves to be an attractive approach to improve the long-term stability of devices. ${ }^{116,166,167}$

Imperfection passivation could also introduce hydrophobic function groups to the perovskite films to improve the stability of PSCs. ${ }^{168} \mathrm{Wu}$ et al. proposed the bilateral alkylamine
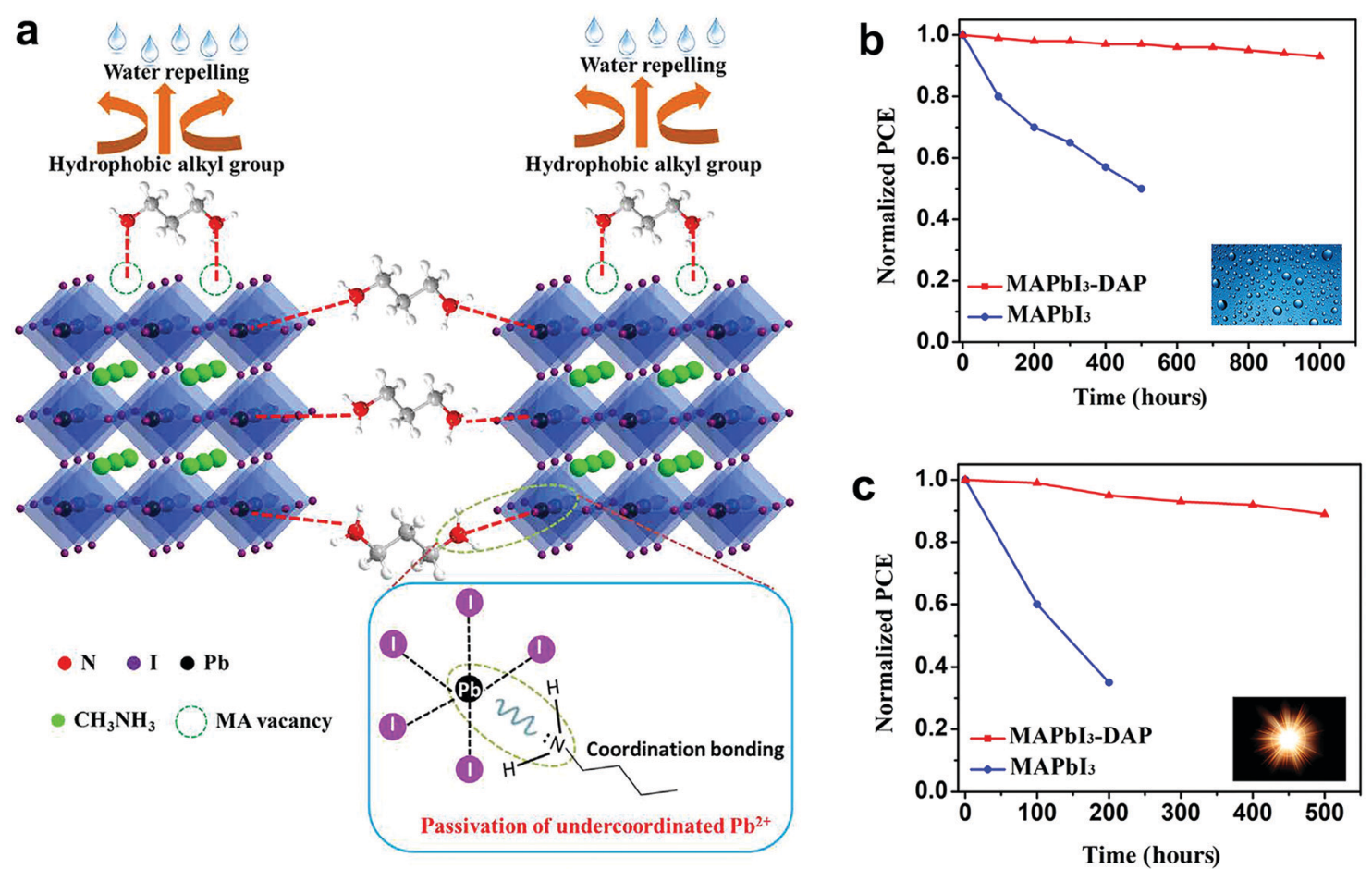

Fig. 12 Improved stability of PSCs by defect passivation. (a) Schematic illustration of defect passivation and water repellence induced by bilateral alkylamine incorporation; (b) moisture stability of un-encapsulated PSCs based on bilateral alkylamine treated MAPbl 3 (MAPb/ ${ }_{3}$-DAP) and pristine MAPbl 3 films under ambient air; (c) stability of encapsulated PSCs based on MAPb/ ${ }_{3}$-DAP and pristine MAPbl 3 films under continuous 1 sun illumination. Reproduced from ref. 168 with permission from the AAAS, copyright 2019. 
additives for perovskite precursor (Fig. 12a), where the hydrophilic $-\mathrm{NH}_{2}$ tails at both ends could form coordinate bonds with undercoordinated $\mathrm{Pb}^{2+}$ or occupy A-site vacancies, leaving the hydrophobic alkyl chain linker exposed to achieve a moistureresistant barrier at the surface and grain boundaries of $\mathrm{MAPbI}_{3}$ films. ${ }^{168}$ The passivation effect of $-\mathrm{NH}_{2}$ tails improves the device $V_{\mathrm{OC}}$ and PCE, and the hydrophobic alkyl chain linker significantly enhances the device stability when exposed to moisture and illumination (Fig. 12b and c). ${ }^{168}$

Moreover, suppression of ion migration could prevent the reaction of mobile ions with the charge transport layers or metal electrode to improve the long-term stability of PSCs. Hermes et al. found that the reaction between oxidized spiro$\mathrm{OMeTAD}^{+}$and mobile $\mathrm{I}^{-}$ions in PSCs can be reduced by replacing the planar $\mathrm{TiO}_{2}$ ETL with a C60-SAM ETL, capable of improved charge extraction. ${ }^{49}$ As a result, the PSCs with a C60-SAM ETL demonstrate better stability relative to their $\mathrm{TiO}_{2}$ based counterparts. Ion migration also results in phase segregation of mixed halide perovskite solar cells, detrimental to device performance and stability. Abdi-Jalebi et al. found this photoinduced phase segregation not only occurs in wide bandgap OIHP materials with $\mathrm{Br}$ content $>20 \%$, but also in commonly used $\left(\mathrm{Cs}_{0.06} \mathrm{FA}_{0.79} \mathrm{MA}_{0.15}\right) \mathrm{Pb}\left(\mathrm{I}_{0.85} \mathrm{Br}_{0.15}\right)_{3}$ perovskite with bandgap of $1.55 \mathrm{eV}{ }^{47}$ Therefore, the inhibition of photoinduced ion migration by passivation of grain boundaries has been found to enhance the long-term stability of mixed-halide PSCs. ${ }^{47}$

\section{Summary and outlook}

Since the recent development of OIHPs and their initial application in photovoltaics, their ever-growing efficiency coupled with low-temperature solution processing and low materials cost promise an exceptional LCOE with a dramatically reduced energetic fabrication debt, ultimately providing an environmentally attractive and economically viable alternative to current commercial photovoltaics. In order to improve the PCE and stability of perovskite solar cells, the imperfection passivation to reduce the non-radiative recombination and suppress the ion migration has proven to be invaluable.

Here, we have given an in-depth review of passivation techniques reported thus far using coordinate bonding and ionic bonding to target the charged defects of the surface and grain boundaries as well as the conversion of those extended defects to wide bandgap materials to produce a favorable type-I heterojunction. Even though the point defects generally form shallow level traps in OIHP materials, which should not contribute to non-radiative recombination processes, the unique ion migration properties of OIHP materials create some pathways for point defects to impact the photovoltaic performance of PSCs. We have also discussed the impact of band bending, interfacial reactions, and phase segregation resulting from the migration of point defects. This highlights the importance of not only passivating extended imperfections but also mitigating the migration of ions and point defects. Additionally, not considered thus far is that when charged defects and trapped charges at the surface are eliminated via passivation, it might mitigate the coulombic forces that normally would repel defects migrating under an electric field. Eliminating coulombic forces from the surface may allow for point defects from the bulk to migrate to the surface, effectively passivating the perovskite crystal bulk via surface passivation. More detailed work about the coupling of bulk defects and surface defects is still needed in the future.

The tremendous role that the passivation of both deep- and shallow-level defect induced traps has played in the recent advances of OIHP device performance and stability is undeniable. However, a complete understanding of passivation mechanisms has yet to be totally resolved, greatly in part due to the possible multifunctional nature of some additives, overall complexity of the systems in question, and the limited experimental techniques. Without the capability to pin-point the various types of defects present, their concentration, and their resulting trap depth, it is difficult to achieve universal highly effective passivation across different OIHP thin film fabrication processes or compositions. This is likely the greatest challenge limiting defect passivation of PSCs. Moving forward, the need for a better understanding of passivation mechanisms to guide additive design, as well as combinations of complementary additives to produce synergetic passivation systems will be crucial to further improve the efficiency and stability of OIHP devices. Continuing to improve the passivation of OIHP materials will facilitate the transition of this technology from bench to market.

\section{Conflicts of interest}

The authors declare no competing interests.

\section{Acknowledgements}

This work is supported by Air Force Office of Scientific Research (AFOSR) (Grant No. A9550-16-1-0299) and Office of Naval Research under award N00014-17-1-2727.

\section{References}

1 W. S. Yang, J. H. Noh, N. J. Jeon, Y. C. Kim, S. Ryu, J. Seo and S. I. Seok, Science, 2015, 348, 1234-1237.

2 W. Chen, Y. Wu, Y. Yue, J. Liu, W. Zhang, X. Yang, H. Chen, E. Bi, I. Ashraful, M. Graetzel and L. Han, Science, 2015, 350, 944-948.

3 https://www.nrel.gov/pv/cell-efficiency.html, accessed April 16, 2019.

4 J. Burschka, N. Pellet, S. J. Moon, R. Humphry-Baker, P. Gao, M. K. Nazeeruddin and M. Gratzel, Nature, 2013, 499, 316-319.

5 M. Z. Liu, M. B. Johnston and H. J. Snaith, Nature, 2013, 501, 395-398.

6 J. Huang, Y. Yuan, Y. Shao and Y. Yan, Nat. Rev. Mater., 2017, 2, 17042. 
7 J. Huang, Y. Shao and Q. Dong, J. Phys. Chem. Lett., 2015, 6, 3218-3227.

8 Z. Li, Y. Zhao, X. Wang, Y. Sun, Z. Zhao, Y. Li, H. Zhou and Q. Chen, Joule, 2018, 2, 1559-1572.

9 M. Cai, Y. Wu, H. Chen, X. Yang, Y. Qiang and L. Han, $A d v$. Sci., 2016, 4, 1600269.

10 J. Werner, A. Walter, E. Rucavado, S.-J. Moon, D. Sacchetto, M. Rienaecker, R. Peibst, R. Brendel, X. Niquille and S. De Wolf, Appl. Phys. Lett., 2016, 109, 233902.

11 S. Albrecht, M. Saliba, J. P. C. Baena, F. Lang, L. Kegelmann, M. Mews, L. Steier, A. Abate, J. Rappich, L. Korte, R. Schlatmann, M. K. Nazeeruddin, A. Hagfeldt, M. Graetzel and B. Rech, Energy Environ. Sci., 2016, 9, 81-88.

12 K. A. Bush, A. F. Palmstrom, Z. S. J. Yu, M. Boccard, R. Cheacharoen, J. P. Mailoa, D. P. McMeekin, R. L. Z. Hoye, C. D. Bailie, T. Leijtens, I. M. Peters, M. C. Minichetti, N. Rolston, R. Prasanna, S. Sofia, D. Harwood, W. Ma, F. Moghadam, H. J. Snaith, T. Buonassisi, Z. C. Holman, S. F. Bent and M. D. McGehee, Nat. Energy, 2017, 2, 17009.

13 F. Sahli, J. Werner, B. A. Kamino, M. Brauninger, R. Monnard, B. Paviet-Salomon, L. Barraud, L. Ding, J. J. D. Leon, D. Sacchetto, G. Cattaneo, M. Despeisse, M. Boccard, S. Nicolay, Q. Jeangros, B. Niesen and C. Ballif, Nat. Mater., 2018, 17, 820-826.

14 Z. J. Yu, M. Leilaeioun and Z. Holman, Nat. Energy, 2016, 1, 16137.

15 G. E. Eperon, T. Leijtens, K. A. Bush, R. Prasanna, T. Green, J. T. W. Wang, D. P. McMeekin, G. Volonakis, R. L. Milot, R. May, A. Palmstrom, D. J. Slotcavage, R. A. Belisle, J. B. Patel, E. S. Parrott, R. J. Sutton, W. Ma, F. Moghadam, B. Conings, A. Babayigit, H. G. Boyen, S. Bent, F. Giustino, L. M. Herz, M. B. Johnston, M. D. McGehee and H. J. Snaith, Science, 2016, 354, 861-865.

16 W. Shockley and H. J. Queisser, J. Appl. Phys., 1961, 32, 510-519.

17 M. L. Agiorgousis, Y.-Y. Sun, H. Zeng and S. Zhang, J. Am. Chem. Soc., 2014, 136, 14570-14575.

18 W.-J. Yin, T. Shi and Y. Yan, Appl. Phys. Lett., 2014, 104, 063903.

19 K. X. Steirer, P. Schulz, G. Teeter, V. Stevanovic, M. Yang,

K. Zhu and J. J. Berry, ACS Energy Lett., 2016, 1, 360-366.

20 A. Walsh, D. O. Scanlon, S. Y. Chen, X. G. Gong and S. H. Wei, Angew. Chem., Int. Ed., 2015, 54, 1791-1794.

21 J. Kim, S. H. Lee, J. H. Lee and K. H. Hong, J. Phys. Chem. Lett., 2014, 5, 1312-1317.

22 J. M. Ball and A. Petrozza, Nat. Energy, 2016, 1, 16149.

23 S. D. Stranks, ACS Energy Lett., 2017, 2, 1515-1525.

24 L. M. Pazos-Outón, T. P. Xiao and E. Yablonovitch, J. Phys. Chem. Lett., 2018, 9, 1703-1711.

25 W. Tress, N. Marinova, O. Inganäs, M. K. Nazeeruddin, S. M. Zakeeruddin and M. Graetzel, Adv. Energy Mater., 2015, 5, 1400812.

26 Y. Shao, Z. Xiao, C. Bi, Y. Yuan and J. Huang, Nat. Commun., 2014, 5, 5784.

27 A. Abate, M. Saliba, D. J. Hollman, S. D. Stranks, K. Wojciechowski, R. Avolio, G. Grancini, A. Petrozza and H. J. Snaith, Nano Lett., 2014, 14, 3247-3254.
28 W. Shockley and W. Read Jr, Phys. Rev., 1952, 87, 835.

29 C. Ran, J. Xu, W. Gao, C. Huang and S. Dou, Chem. Soc. Rev., 2018, 47, 4581-4610.

30 K. Domanski, J.-P. Correa-Baena, N. Mine, M. K. Nazeeruddin, A. Abate, M. Saliba, W. Tress, A. Hagfeldt and M. Grätzel, ACS Nano, 2016, 10, 6306-6314.

31 Z. Li, C. X. Xiao, Y. Yang, S. P. Harvey, D. H. Kim, J. A. Christians, M. J. Yang, P. Schulz, S. U. Nanayakkara, C. S. Jiang, J. M. Luther, J. J. Berry, M. C. Beard, M. M. AlJassim and K. Zhu, Energy Environ. Sci., 2017, 10, 1234-1242.

32 X. Wu, M. T. Trinh, D. Niesner, H. Zhu, Z. Norman, J. S. Owen, O. Yaffe, B. J. Kudisch and X.-Y. Zhu, J. Am. Chem. Soc., 2015, 137, 2089-2096.

33 R. Long, J. Liu and O. V. Prezhdo, J. Am. Chem. Soc., 2016, 138, 3884-3890.

34 G. Sadoughi, D. E. Starr, E. Handick, S. D. Stranks, M. Gorgoi, R. G. Wilks, M. Bär and H. J. Snaith, ACS Appl. Mater. Interfaces, 2015, 7, 13440-13444.

35 W. Zhang, S. Pathak, N. Sakai, T. Stergiopoulos, P. K. Nayak, N. K. Noel, A. A. Haghighirad, V. M. Burlakov, A. Sadhanala and W. Li, Nat. Commun., 2015, 6, 10030.

36 Y. Fang, Q. Dong, Y. Shao, Y. Yuan and J. Huang, Nat. Photonics, 2015, 9, 679-686.

37 W. Tress, N. Marinova, T. Moehl, S. M. Zakeeruddin, M. K. Nazeeruddin and M. Graetzel, Energy Environ. Sci., 2015, 8, 995-1004.

38 B. Chen, M. Yang, S. Priya and K. Zhu, J. Phys. Chem. Lett., 2016, 7, 905-917.

39 J. M. Azpiroz, E. Mosconi, J. Bisquert and F. De Angelis, Energy Environ. Sci., 2015, 8, 2118-2127.

40 C. Eames, J. M. Frost, P. R. F. Barnes, B. C. O'Regan, A. Walsh and M. S. Islam, Nat. Commun., 2015, 6, 7497.

41 Y. Yuan and J. Huang, Acc. Chem. Res., 2016, 49, 286-293. 42 J. Xing, Q. Wang, Q. F. Dong, Y. B. Yuan, Y. J. Fanga and J. S. Huang, Phys. Chem. Chem. Phys., 2016, 18, 30484-30490.

43 D. H. Kang and N. G. Park, Adv. Mater., 2019, 1805214.

44 E. T. Hoke, D. J. Slotcavage, E. R. Dohner, A. R. Bowring, H. I. Karunadasa and M. D. McGehee, Chem. Sci., 2015, 6, 613-617.

45 C. G. Bischak, C. L. Hetherington, H. Wu, S. Aloni, D. F. Ogletree, D. T. Limmer and N. S. Ginsberg, Nano Lett., 2017, 17, 1028-1033.

46 D. J. Slotcavage, H. I. Karunadasa and M. D. McGehee, ACS Energy Lett., 2016, 1, 1199-1205.

47 M. Abdi-Jalebi, Z. Andaji-Garmaroudi, S. Cacovich, C. Stavrakas, B. Philippe, J. M. Richter, M. Alsari, E. P. Booker, E. M. Hutter, A. J. Pearson, S. Lilliu, T. J. Savenije, H. Rensmo, G. Divitini, C. Ducati, R. H. Friend and S. D. Stranks, Nature, 2018, 555, 497-501.

48 J. Carrillo, A. Guerrero, S. Rahimnejad, O. Almora, I. Zarazua, E. Mas-Marza, J. Bisquert and G. GarciaBelmonte, Adv. Energy Mater., 2016, 6, 1502246.

49 I. M. Hermes, Y. Hou, V. W. Bergmann, C. J. Brabec and S. A. Weber, J. Phys. Chem. Lett., 2018, 9, 6249-6256. 
50 S. Kim, S. Bae, S.-W. Lee, K. Cho, K. D. Lee, H. Kim, S. Park, G. Kwon, S.-W. Ahn and H.-M. Lee, Sci. Rep., 2017, 7, 1200. 51 S. Wu, R. Chen, S. Zhang, B. H. Babu, Y. Yue, H. Zhu, Z. Yang, C. Chen, W. Chen, Y. Huang, S. F. Fang, T. Liu, L. Han and W. Chen, Nat. Commun., 2019, 10, 1161.

52 Y. Kato, L. K. Ono, M. V. Lee, S. Wang, S. R. Raga and Y. Qi, Adv. Mater. Interfaces, 2015, 2, 1500195.

53 R. T. Ross, J. Chem. Phys., 1967, 46, 4590-4593.

54 T. S. Sherkar, C. Momblona, L. Gil-Escrig, J. Ávila, M. Sessolo, H. J. Bolink and L. J. A. Koster, ACS Energy Lett., 2017, 2, 1214-1222.

55 J. Xu, A. Buin, A. H. Ip, W. Li, O. Voznyy, R. Comin, M. Yuan, S. Jeon, Z. Ning, J. J. McDowell, P. Kanjanaboos, J.-P. Sun, X. Lan, L. N. Quan, D. H. Kim, I. G. Hill, P. Maksymovych and E. H. Sargent, Nat. Commun., 2015, 6, 7081.

56 A. G. Aberle, Sol. Energy Mater. Sol. Cells, 2001, 65, 239-248. 57 C. Bi, X. Zheng, B. Chen, H. Wei and J. Huang, ACS Energy Lett., 2017, 2, 1400-1406.

58 M. Jung, T. J. Shin, J. Seo, G. Kim and S. I. Seok, Energy Environ. Sci., 2018, 11, 2188-2197.

59 Y. Fu, T. Wu, J. Wang, J. Zhai, M. J. Shearer, Y. Zhao, R. J. Hamers, E. Kan, K. Deng, X.-Y. Zhu and S. Jin, Nano Lett., 2017, 17, 4405-4414.

60 D.-Y. Son, J.-W. Lee, Y. J. Choi, I.-H. Jang, S. Lee, P. J. Yoo, H. Shin, N. Ahn, M. Choi, D. Kim and N.-G. Park, Nat. Energy, 2016, 1, 16081.

61 B. Hoex, J. Schmidt, P. Pohl, M. Van de Sanden and W. Kessels, J. Appl. Phys., 2008, 104, 044903.

62 A. G. Aberle, Prog. Photovoltaics Res. Appl., 2000, 8, 473-487.

63 Y. B. Yuan, T. Li, Q. Wang, J. Xing, A. Gruverman and J. S. Huang, Sci. Adv., 2017, 3, e1602164.

64 Y. C. Shao, Y. J. Fang, T. Li, Q. Wang, Q. F. Dong, Y. H. Deng, Y. B. Yuan, H. T. Wei, M. Y. Wang, A. Gruverman, J. Shielda and J. S. Huang, Energy Environ. Sci., 2016, 9, 1752-1759.

65 J. Chen and N. G. Park, Adv. Mater., 2018, 1803019.

66 F. Wang, S. Bai, W. Tress, A. Hagfeldt and F. Gao, npj Flex. Electron., 2018, 2, 22.

67 D. Bi, W. Tress, M. I. Dar, P. Gao, J. Luo, C. Renevier, K. Schenk, A. Abate, F. Giordano and J.-P. C. Baena, Sci. Adv., 2016, 2, e1501170.

68 Q. Chen, H. Zhou, T.-B. Song, S. Luo, Z. Hong, H.-S. Duan, L. Dou, Y. Liu and Y. Yang, Nano Lett., 2014, 14, 4158-4163.

69 C. Bi, Y. C. Shao, Y. B. Yuan, Z. G. Xiao, C. G. Wang, Y. L. Gao and J. S. Huang, J. Mater. Chem. A, 2014, 2, 18508-18514.

70 A. Dualeh, N. Tétreault, T. Moehl, P. Gao, M. K. Nazeeruddin and M. Grätzel, Adv. Funct. Mater., 2014, 24, 3250-3258.

71 M. I. Saidaminov, J. Kim, A. Jain, R. Quintero-Bermudez, H. R. Tan, G. K. Long, F. R. Tan, A. Johnston, Y. C. Zhao, O. Voznyy and E. H. Sargent, Nat. Energy, 2018, 3, 648-654.

72 L. K. Ono and Y. Qi, J. Phys. Chem. Lett., 2016, 7, 4764-4794.

73 L. She, M. Liu and D. Zhong, ACS Nano, 2015, 10, 1126-1131.
74 R. Ohmann, L. K. Ono, H.-S. Kim, H. Lin, M. V. Lee, Y. Li, N.-G. Park and Y. Qi, J. Am. Chem. Soc., 2015, 137, 16049-16054.

75 A. Baumann, S. Väth, P. Rieder, M. C. Heiber, K. Tvingstedt and V. Dyakonov, J. Phys. Chem. Lett., 2015, 6, 2350-2354.

76 S. Heo, G. Seo, Y. Lee, D. Lee, M. Seol, J. Lee, J.-B. Park, K. Kim, D.-J. Yun and Y. S. Kim, Energy Environ. Sci., 2017, 10, 1128-1133.

77 G. Niu, X. Guo and L. Wang, J. Mater. Chem. A, 2015, 3, 8970-8980.

78 R. Lindblad, D. Bi, B.-w. Park, J. Oscarsson, M. Gorgoi, H. Siegbahn, M. Odelius, E. M. Johansson and H. k. Rensmo, J. Phys. Chem. Lett., 2014, 5, 648-653.

79 S. Wang, Y. Jiang, E. J. Juarez-Perez, L. K. Ono and Y. Qi, Nat. Energy, 2017, 2, 16195.

80 Y. Lin, B. Chen, F. Zhao, X. Zheng, Y. Deng, Y. Shao, Y. Fang, Y. Bai, C. Wang and J. Huang, Adv. Mater., 2017, 29, 1700607.

81 R. Haddon, Philos. Trans. R. Soc., A, 1993, 343, 53-62.

82 H.-H. Fang, S. Adjokatse, H. Wei, J. Yang, G. R. Blake, J. Huang, J. Even and M. A. Loi, Sci. Adv., 2016, 2, e1600534.

83 Y. Tian, M. Peter, E. Unger, M. Abdellah, K. Zheng, T. Pullerits, A. Yartsev, V. Sundström and I. G. Scheblykin, Phys. Chem. Chem. Phys., 2015, 17, 24978-24987.

84 S. Yang, Y. Wang, P. Liu, Y.-B. Cheng, H. J. Zhao and H. G. Yang, Nat. Energy, 2016, 1, 15016.

85 N. Aristidou, C. Eames, I. Sanchez-Molina, X. Bu, J. Kosco, M. S. Islam and S. A. Haque, Nat. Commun., 2017, 8, 15218.

86 G. E. Eperon, S. N. Habisreutinger, T. Leijtens, B. J. Bruijnaers, J. J. van Franeker, D. W. deQuilettes, S. Pathak, R. J. Sutton, G. Grancini and D. S. Ginger, ACS Nano, 2015, 9, 9380-9393.

87 H. Zhou, Q. Chen, G. Li, S. Luo, T.-B. Song, H.-S. Duan, Z. Hong, J. You, Y. Liu and Y. Yang, Science, 2014, 345, 542-546.

88 D. Meggiolaro, E. Mosconi and F. De Angelis, ACS Energy Lett., 2017, 2, 2794-2798.

89 F. De Angelis and A. Petrozza, Nat. Mater., 2018, 17, 383-384.

90 N. K. Noel, A. Abate, S. D. Stranks, E. S. Parrott, V. M. Burlakov, A. Goriely and H. J. Snaith, ACS Nano, 2014, 8, 9815-9821.

91 K. Wojciechowski, T. Leijtens, S. Siprova, C. Schlueter, M. T. Hörantner, J. T.-W. Wang, C.-Z. Li, A. K.-Y. Jen, T.-L. Lee and H. J. Snaith, J. Phys. Chem. Lett., 2015, 6, 2399-2405.

92 J. Cao, J. Yin, S. Yuan, Y. Zhao, J. Li and N. Zheng, Nanoscale, 2015, 7, 9443-9447.

93 W. Li, H. Dong, X. Guo, N. Li, J. Li, G. Niu and L. Wang, J. Mater. Chem. A, 2014, 2, 20105-20111.

94 F. Wang, W. Geng, Y. Zhou, H. H. Fang, C. J. Tong, M. A. Loi, L. M. Liu and N. Zhao, Adv. Mater., 2016, 28, 9986-9992.

95 Y. Lin, L. Shen, J. Dai, Y. Deng, Y. Wu, Y. Bai, X. Zheng, J. Wang, Y. Fang and H. Wei, Adv. Mater., 2017, 29, 1604545. 
96 M. Hadadian, J. P. Correa-Baena, E. K. Goharshadi, A. Ummadisingu, J. Y. Seo, J. Luo, S. Gholipour, S. M. Zakeeruddin, M. Saliba and A. Abate, Adv. Mater., 2016, 28, 8681-8686.

97 L. Zuo, H. Guo, S. Jariwala, N. De Marco, S. Dong, R. DeBlock, D. S. Ginger, B. Dunn, M. Wang and Y. Yang, Sci. Adv., 2017, 3, e1700106.

98 F. Palazon, D. Pérez-del-Rey, S. Marras, M. Prato, M. Sessolo, H. J. Bolink and L. Manna, ACS Energy Lett., 2018, 3, 835-839.

99 T. Y. Wen, S. Yang, P. F. Liu, L. J. Tang, H. W. Qiao, X. Chen, X. H. Yang, Y. Hou and H. G. Yang, Adv. Energy Mater., 2018, 8, 1703143.

100 Q. Zeng, X. Zhang, X. Feng, S. Lu, Z. Chen, X. Yong, S. A. T. Redfern, H. Wei, H. Wang, H. Shen, W. Zhang, W. Zheng, H. Zhang, J. S. Tse and B. Yang, Adv. Mater., 2018, 30, 1705393.

101 L. Liu, W.-H. Fang, R. Long and O. V. Prezhdo, J. Phys. Chem. Lett., 2018, 9, 1164-1171.

102 D. W. deQuilettes, S. Koch, S. Burke, R. K. Paranji, A. J. Shropshire, M. E. Ziffer and D. S. Ginger, ACS Energy Lett., 2016, 1, 438-444.

103 I. L. Braly, D. W. deQilettes, L. M. Pazos-Outon, S. Burke, M. E. Ziffer, D. S. Ginger and H. W. Hillhouse, Nat. Photonics, 2018, 12, 355-361.

104 K. S. Novoselov, V. Fal, L. Colombo, P. Gellert, M. Schwab and K. Kim, Nature, 2012, 490, 192.

105 Z. Wu, S. Bai, J. Xiang, Z. Yuan, Y. Yang, W. Cui, X. Gao, Z. Liu, Y. Jin and B. Sun, Nanoscale, 2014, 6, 10505-10510.

106 S. Lee, J. H. Park, B. R. Lee, E. D. Jung, J. C. Yu, D. Di Nuzzo, R. H. Friend and M. H. Song, J. Phys. Chem. Lett., 2017, 8, 1784-1792.

107 D. J. Kubicki, D. Prochowicz, A. Hofstetter, S. M. Zakeeruddin, M. Grätzel and L. Emsley, J. Am. Chem. Soc., 2017, 139, 14173-14180.

108 D.-Y. Son, S.-G. Kim, J.-Y. Seo, S.-H. Lee, H. Shin, D. Lee and N.-G. Park, J. Am. Chem. Soc., 2018, 140, 1358-1364.

109 M. Abdi-Jalebi, M. I. Dar, A. Sadhanala, S. P. Senanayak, M. Franckevičius, N. Arora, Y. Hu, M. K. Nazeeruddin, S. M. Zakeeruddin, M. Grätzel and R. H. Friend, $A d v$. Energy Mater., 2016, 6, 1502472.

110 M. Saliba, T. Matsui, K. Domanski, J. Y. Seo, A. Ummadisingu, S. M. Zakeeruddin, J. P. Correa-Baena, W. R. Tress, A. Abate, A. Hagfeldt and M. Gratzel, Science, 2016, 354, 206-209.

111 P. Zhao, W. Yin, M. Kim, M. Han, Y. J. Song, T. K. Ahn and H. S. Jung, J. Mater. Chem. A, 2017, 5, 7905-7911.

112 M. Abdi-Jalebi, M. Pazoki, B. Philippe, M. I. Dar, M. Alsari, A. Sadhanala, G. Diyitini, R. Imani, S. Lilliu, J. Kullgren, H. Rensmo, M. Gratzel and R. H. Friend, ACS Nano, 2018, 12, 7301-7311.

113 M. Zhang, J. S. Yun, Q. S. Ma, J. H. Zheng, C. F. J. Lau, X. F. Deng, J. Kim, D. Kim, J. Seidel, M. A. Green, S. J. Huang and A. W. Y. Ho-Baillie, ACS Energy Lett., 2017, 2, 438-444.

114 T. Zhao, C.-C. Chueh, Q. Chen, A. Rajagopal and A. K.-Y. Jen, ACS Energy Lett., 2016, 1, 757-763.
115 E. Jokar, C.-H. Chien, Y.-H. Chang, A. Fathi, M. Rameez and E. W.-G. Diau, Energy Environ. Sci., 2018, 11, 2353-2362.

116 Y. Lin, Y. Bai, Y. Fang, Z. Chen, S. Yang, X. Zheng, S. Tang, Y. Liu, J. Zhao and J. Huang, J. Phys. Chem. Lett., 2018, 9, 654-658.

117 Z. P. Wang, Q. Q. Lin, F. P. Chmiel, N. Sakai, L. M. Herz and H. J. Snaith, Nat. Energy, 2017, 2, 17135.

118 D. S. Lee, J. S. Yun, J. Kim, A. M. Soufiani, S. Chen, Y. Cho, X. Deng, J. Seidel, S. Lim and S. Huang, ACS Energy Lett., 2018, 3, 647-654.

119 G. Grancini, C. Roldan-Carmona, I. Zimmermann, E. Mosconi, X. Lee, D. Martineau, S. Narbey, F. Oswald, F. De Angelis, M. Graetzel and M. K. Nazeeruddin, Nat. Commun., 2017, 8, 15684.

120 Z. Y. Cheng and J. Lin, CrystEngComm, 2010, 12, 2646-2662.

121 I. C. Smith, E. T. Hoke, D. Solis-Ibarra, M. D. McGehee and H. I. Karunadasa, Angew. Chem., 2014, 126, 11414-11417.

122 Q. Jiang, Y. Zhao, X. Zhang, X. Yang, Y. Chen, Z. Chu, Q. Ye, X. Li, Z. Yin and J. You, Nat. Photonics, 2019, DOI: 10.1038/s41566-019-0398-2.

123 Z. Wang, Y. Zhou, S. Pang, Z. Xiao, J. Zhang, W. Chai, H. Xu, Z. Liu, N. P. Padture and G. Cui, Chem. Mater., 2015, 27, 7149-7155.

124 B. Chen, Z. Yu, K. Liu, X. Zheng, Y. Liu, J. Shi, D. Spronk, P. N. Rudd, Z. Holman and J. Huang, Joule, 2019, 3, 177-190.

125 J. I. Uribe, J. Ciro, J. F. Montoya, J. Osorio and F. Jaramillo, ACS Appl. Energy Mater., 2018, 1, 1047-1052.

126 F. Xie, C.-C. Chen, Y. Wu, X. Li, M. Cai, X. Liu, X. Yang and L. Han, Energy Environ. Sci., 2017, 10, 1942-1949.

127 Q. Li, Y. Zhao, R. Fu, W. Zhou, Y. Zhao, X. Liu, D. Yu and Q. Zhao, Adv. Mater., 2018, 30, 1803095.

128 M. M. Lee, J. Teuscher, T. Miyasaka, T. N. Murakami and H. J. Snaith, Science, 2012, 338, 643-647.

129 V. L. Pool, A. Gold-Parker, M. D. McGehee and M. F. Toney, Chem. Mater., 2015, 27, 7240-7243.

130 N. Yantara, F. Yanan, C. Shi, H. A. Dewi, P. P. Boix, S. G. Mhaisalkar and N. Mathews, Chem. Mater., 2015, 27, 2309-2314.

131 Y. Q. Luo, S. Gamliel, S. Nijem, S. Aharon, M. Holt, B. Stripe, V. Rose, M. I. Bertoni, L. Etgar and D. P. Fenning, Chem. Mater., 2016, 28, 6536-6543.

132 Q. Chen, H. Zhou, Y. Fang, A. Z. Stieg, T.-B. Song, H.-H. Wang, X. Xu, Y. Liu, S. Lu and J. You, Nat. Commun., 2015, 6, 7269 .

133 G. J. Nan, X. Zhang, M. Abdi-Jalebi, Z. Andaji-Garmaroudi, S. D. Stranks, G. Lu and D. Beljonne, Adv. Energy Mater., 2018, 8, 1702754.

134 H. R. Tan, A. Jain, O. Voznyy, X. Z. Lan, F. P. G. de Arquer, J. Z. Fan, R. Quintero-Bermudez, M. J. Yuan, B. Zhang, Y. C. Zhao, F. J. Fan, P. C. Li, L. N. Quan, Y. B. Zhao, Z. H. Lu, Z. Y. Yang, S. Hoogland and E. H. Sargent, Science, 2017, 355, 722-726.

135 X. Li, C. C. Chen, M. L. Cai, X. Hua, F. X. Xie, X. Liu, J. L. Hua, Y. T. Long, H. Tian and L. Y. Han, Adv. Energy Mater., 2018, 8, 1800715. 
136 S. Yang, J. Dai, Z. Yu, Y. Shao, Y. Zhou, X. Xiao, X. C. Zeng and J. Huang, J. Am. Chem. Soc., 2019, 141, 5781-5787.

137 Y. Hu, J. Schlipf, M. Wussler, M. L. Petrus, W. Jaegermann, T. Bein, P. Müller-Buschbaum and P. Docampo, ACS Nano, 2016, 10, 5999-6007.

138 A. Rajagopal, R. J. Stoddard, S. B. Jo, H. W. Hillhouse and A. K.-Y. Jen, Nano Lett., 2018, 18, 3985-3993.

139 X. P. Zheng, B. Chen, J. Dai, Y. J. Fang, Y. Bai, Y. Z. Lin, H. T. Wei, X. C. Zeng and J. S. Huang, Nat. Energy, 2017, 2, 17102.

140 X. Zheng, Y. Deng, B. Chen, H. Wei, X. Xiao, Y. Fang, Y. Lin, Z. Yu, Y. Liu, Q. Wang and J. Huang, Adv. Mater., 2018, 30, 1803428.

141 Q. Wang, X. Zheng, Y. Deng, J. Zhao, Z. Chen and J. Huang, Joule, 2017, 1, 371-382.

142 D. Wei, F. S. Ma, R. Wang, S. Y. Dou, P. Cui, H. Huang, J. Ji, E. D. Jia, X. J. Jia, S. Sajid, A. M. Eiseman, L. H. Chu, Y. F. Li, B. Jiang, J. Qiao, Y. B. Yuan and M. C. Li, $A d v$. Mater., 2018, 30, 1707583.

143 P. L. Qin, J. L. Zhang, G. Yang, X. L. Yu and G. Li, J. Mater. Chem. A, 2019, 7, 1824-1834.

144 N. Li, Z. Zhu, C.-C. Chueh, H. Liu, B. Peng, A. Petrone, X. Li, L. Wang and A. K.-Y. Jen, Adv. Energy Mater., 2017, 7, 1601307.

145 Y. Yuan, J. Chae, Y. Shao, Q. Wang, Z. Xiao, A. Centrone and J. Huang, Adv. Energy Mater., 2015, 5, 1500615.

146 Y. Yuan, Q. Wang, Y. Shao, H. Lu, T. Li, A. Gruverman and J. Huang, Adv. Energy Mater., 2016, 6, 1501803.

147 D. W. deQuilettes, W. Zhang, V. M. Burlakov, D. J. Graham, T. Leijtens, A. Osherov, V. Bulovic, H. J. Snaith, D. S. Ginger and S. D. Stranks, Nat. Commun., 2016, 7, 11683.

148 Y.-C. Zhao, W.-K. Zhou, X. Zhou, K.-H. Liu, D.-P. Yu and Q. Zhao, Light: Sci. Appl., 2017, 6, e16243.

149 Z. Xiao, Y. Yuan, Y. Shao, Q. Wang, Q. Dong, C. Bi, P. Sharma, A. Gruverman and J. Huang, Nat. Mater., 2015, 14, 193-198.

150 Y. H. Deng, Z. G. Xiao and J. S. Huang, Adv. Energy Mater., 2015, 5, 1500721.

151 H. Yu, H. Lu, F. Xie, S. Zhou and N. Zhao, Adv. Funct. Mater., 2016, 26, 1411-1419.

152 Y. Lin, B. Chen, Y. Fang, J. Zhao, C. Bao, Z. Yu, Y. Deng, P. N. Rudd, Y. Yan, Y. Yuan and J. Huang, Nat. Commun., 2018, 9, 4981.

153 S. T. Birkhold, J. T. Precht, H. B. Liu, R. Giridharagopal, G. E. Eperon, L. Schmidt-Mende, X. S. Li and D. S. Ginger, ACS Energy Lett., 2018, 3, 1279-1286.
154 F. Giordano, A. Abate, J. P. C. Baena, M. Saliba, T. Matsui, S. H. Im, S. M. Zakeeruddin, M. K. Nazeeruddin, A. Hagfeldt and M. Graetzel, Nat. Commun., 2016, 7, 10379.

155 Q. Jiang, L. Q. Zhang, H. L. Wang, X. L. Yang, J. H. Meng, H. Liu, Z. G. Yin, J. L. Wu, X. W. Zhang and J. B. You, Nat. Energy, 2017, 2, 16177.

156 N.-G. Park, M. Grätzel, T. Miyasaka, K. Zhu and K. Emery, Nat. Energy, 2016, 1, 16152.

157 A. l. M. Leguy, Y. Hu, M. Campoy-Quiles, M. I. Alonso, O. J. Weber, P. Azarhoosh, M. Van Schilfgaarde, M. T. Weller, T. Bein and J. Nelson, Chem. Mater., 2015, 27, 3397-3407.

158 N. Ahn, K. Kwak, M. S. Jang, H. Yoon, B. Y. Lee, J.-K. Lee, P. V. Pikhitsa, J. Byun and M. Choi, Nat. Commun., 2016, 7, 13422.

159 N. Aristidou, I. Sanchez-Molina, T. Chotchuangchutchaval, M. Brown, L. Martinez, T. Rath and S. A. Haque, Angew. Chem., 2015, 127, 8326-8330.

160 A. J. Pearson, G. E. Eperon, P. E. Hopkinson, S. N. Habisreutinger, J. T. W. Wang, H. J. Snaith and N. C. Greenham, Adv. Energy Mater., 2016, 6, 1600014.

161 D. Bryant, N. Aristidou, S. Pont, I. Sanchez-Molina, T. Chotchunangatchaval, S. Wheeler, J. R. Durrant and S. A. Haque, Energy Environ. Sci., 2016, 9, 1655-1660.

162 Q. Wang, B. Chen, Y. Liu, Y. Deng, Y. Bai, Q. Dong and J. Huang, Energy Environ. Sci., 2017, 10, 516-522.

163 X. Zhang, X. D. Ren, B. Liu, R. Munir, X. J. Zhu, D. Yang, J. B. Li, Y. C. Liu, D. M. Smilgies, R. P. Li, Z. Yang, T. Q. Niu, X. L. Wang, A. Amassian, K. Zhao and S. Z. F. Liu, Energy Environ. Sci., 2017, 10, 2095-2102.

164 L. N. Quan, M. Yuan, R. Comin, O. Voznyy, E. M. Beauregard, S. Hoogland, A. Buin, A. R. Kirmani, K. Zhao, A. Amassian, D. H. Kim and E. H. Sargent, J. Am. Chem. Soc., 2016, 138, 2649-2655.

165 T. Y. Zhang, L. Q. Xie, L. Chen, N. J. Guo, G. Li, Z. Q. Tian, B. W. Mao and Y. X. Zhao, Adv. Funct. Mater., 2017, 27, 1603568.

166 Y. Lin, Y. Bai, Y. Fang, Q. Wang, Y. Deng and J. Huang, ACS Energy Lett., 2017, 2, 1571-1572.

167 Z. Wang, Q. Lin, F. P. Chmiel, N. Sakai, L. M. Herz and H. J. Snaith, Nature, 2017, 2, 17135.

168 W.-Q. Wu, Z. Yang, P. N. Rudd, Y. Shao, X. Dai, H. Wei, J. Zhao, Y. Fang, Q. Wang, Y. Liu, Y. Deng, X. Xiao, Y. Feng and J. Huang, Sci. Adv., 2019, 5, eaav8925. 